\title{
Suzuki-aza-Wittig, Suzuki-condensation and aza-Wittig-electrocyclic ring-closure tandem reactions for synthesis of fused nitrogen-containing ring systems
}

\author{
Gábor Krajsovszky, László Károlyházy, Petra Dunkel, Sándor Boros, \\ Antonino Grillo, and Péter Mátyus* \\ Department of Organic Chemistry, Semmelweis University, Högyes E. u. 7, \\ H-1092 Budapest, Hungary \\ E-mail: peter.matyus@szerves.sote.hu
}

\begin{abstract}
We describe tandem combinations of Suzuki-aza-Wittig, Suzuki-condensation and aza-Wittigelectrocyclic ring closure reactions for the synthesis of new pyridazino[4,5-c]isoquinolinone, pyridazino[4,5-c]quinolinone and pyrimido[5,4-c]quinoline derivatives.
\end{abstract}

Keywords: Halopyridazin-3(2H)-one, Suzuki reaction, aza-Wittig reaction, tandem reaction, iminophosphoranes

\section{Introduction}

In our previous studies we reported the synthesis of several new polycyclic pyridazines via interand intramolecular nucleophilic substitutions ${ }^{1}$ as well as by using Pd-catalyzed reactions. ${ }^{2}$ In this paper the application of Suzuki-aza-Wittig tandem, Suzuki-condensation (ring closure) tandem, as well as aza-Wittig-electrocyclization tandem reactions are described. The aza-Wittig reaction ${ }^{3}$ is a widely used preparative method for the synthesis of several types of organic compounds.

Synthesis of the pyridazino[4,5-c]isoquinoline ring system from an isoquinoline precursor is well known in the literature. ${ }^{4}$ In our recent studies we showed another route, ${ }^{5}$ in which pyridazino[4,5-c]isoquinoline derivatives 4 and 7 were synthesized by Suzuki cross-coupling reactions from 4-chloro-5-methoxy pyridazin-3(2H)-ones, ${ }^{6}$ and 5-chloro-4-methoxypyridazin$3(2 \mathrm{H})$-ones, ${ }^{7}$ respectively (Scheme 1 ). Now, on the basis of our previous experiences we were interested in new synthetic routes consisting of halogen $\rightarrow$ nitrogen displacements on the pyridazinone ring system with subsequent ring closure reactions. Incorporation of the nitrogen atom into the target ring was carried out via transformation of dihalopyridazinones into haloiminophosphoranes, or haloamine intermediates. 
On the other hand, we elaborated a synthetic method towards 4,5-annelated diazinones via carbodiimides. The synthesis of fused pyridazinones started from the appropriate azides ${ }^{8}$. The basis of this procedure is the conversion of aryliminophosphoranes to carbodiimides by using phenylisocyanate. Iminophosphorane derivatives of pyridazinones ${ }^{9}$ and uracil ${ }^{10}$ are known in the literature, as well as the methodology for preparation of carbodiimides. ${ }^{11}$ Appropriate diarylcarbodiimide can be subjected to ring closure reaction by refluxing in dry solvent, usually toluene. ${ }^{12}$ In our present work this commonly used reaction sequence was applied for the conversion of diazines.

The pyridazino[4,5-c] quinoline ring system has been earlier described in the literature, ${ }^{13}$ where the pyridazine part was built up on a quinoline moety. However, in our synthetic strategy the target ring system was obtained starting from a pyridazine nucleus.

Electrocyclization reaction of an uracil carbodiimide was carried out for the synthesis of pyrimido[5,4-c] quinoline derivatives.

\section{Results and Discussion}

We have published two general methods for the incorporation of a nitrogen atom into a polycyclic system. The first method involved the formation of an azide intermediate, ${ }^{8,14}$ while the second one was realized via a halogen atom displacement of dihalopyridazine by an amino group $^{15,16}$ (Scheme 1).

The starting 2-alkyl-4,5-dibromopyridazin-3(2H)-ones 1a-c, were synthesized according the literature via alkylation of dichloro-,${ }^{17}$ dibromo-,${ }^{18}$ or diiodopyridazin-3(2H)-one. ${ }^{19}$ Compound 1d was obtained similarly, while the literature described its synthesis by the reaction of the 2methyldichloro compound $\mathbf{1 c}$ with concentrated HI. ${ }^{20}$

Regioselective amination of 4,5-dibromo-2-methylpyridazin-3(2H)-one $\mathbf{1 b},{ }^{21}$ and 2-benzyl4,5-dibromopyridazin-3(2H)-one $\mathbf{1 a}^{22}$ could not be achieved by reaction with aqueous ammonia to the appropriate 5-amino-4-bromo derivatives $\mathbf{5} \mathbf{a}^{23}$ and $\mathbf{5} \mathbf{b}^{21}$ as the isomeric 4-amino-5-bromo compounds $\mathbf{6 a}$ and $\mathbf{6} \mathbf{b}^{21}$ were also formed in minor amounts. The same problem was encountered in the case of the published ${ }^{15,16}$ reactions of 4,5-dichloro-2-methylpyridazin-3(2H)-one $1 \mathbf{c}^{17}$ with different amines. On the contrary, $\mathbf{2 a}, \mathbf{2 b},{ }^{24} \mathbf{2 c}^{24}$ and $\mathbf{2 d}$ i.e. the azido derivatives of $\mathbf{1 a - d}$ were obtained selectively from the appropriate dihalopyridazinones by reaction with sodium azide. Reduction of the azides to amines could be problematic; however, the formation of iminophosphorane from the azides could serve as another possibility. 

a : $\mathrm{R}=\mathrm{Bn}, \mathrm{X}=\mathrm{Br}$
b : $\mathrm{R}=\mathrm{Me}, \mathrm{X}=\mathrm{Br}$
c: $\mathrm{R}=\mathrm{Me}, \mathrm{X}=\mathrm{Cl}$
d : $\mathrm{R}=\mathrm{Me}, \mathrm{X}=\mathrm{I}$<smiles>[X]c1cnn([R])c(=O)c1[X]</smiles>

Bn: benzyl

Me: methyl

DME: dimethoxyethane

$$
\text { d }
$$

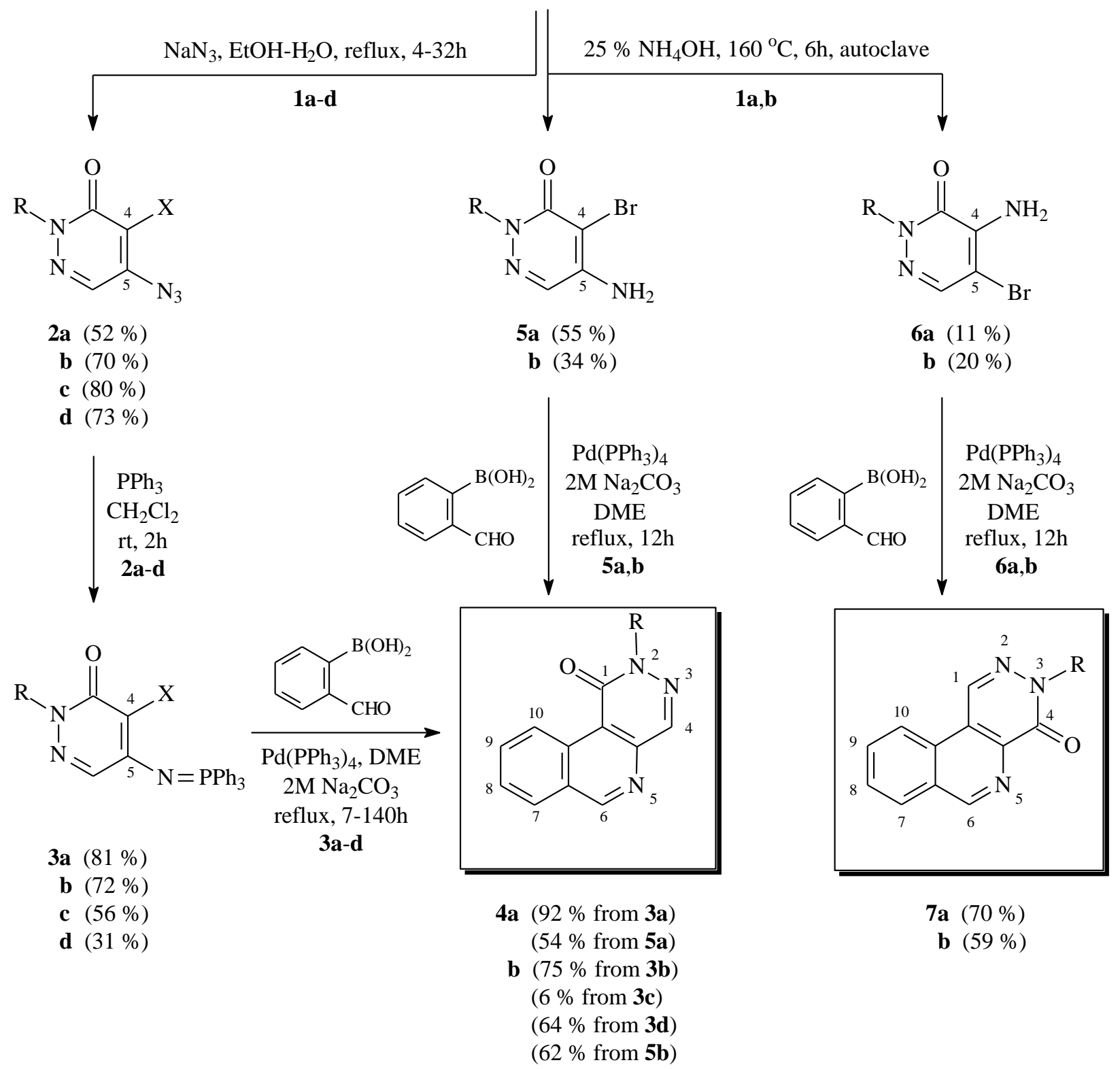

Scheme 1. Suzuki-aza-Wittig and Suzuki-condensation tandem reactions of pyridazinones. 


\section{Suzuki-aza-Wittig tandem reaction}

Iminophosphorane derivatives of pyridazine ${ }^{9}$ and uracil $^{10}$ are well known in the literature. The 4halo-5-iminophosphoranes 3a-d were prepared from the reaction of the related azides with triphenylphosphine followed by the reaction with 2-formylphenylboronic acid to obtain pyridazino[4,5-c]isoquinolin-1(2H)-ones 4a,b (Scheme 1).

Iminophosphoranes 3a-d, which contain different halogen atoms at position 4 , were produced in various yields: iodo derivative 3d was obtained with the lowest yield (31\%), probably as a consequence of steric hindrance. Suzuki cross coupling - condensation tandem reaction has taken place with lowest yield (6\%) when starting with chloroiminophosphorane 3c, as the increasing reactivity order of halogens in Suzuki reaction is $\mathrm{Cl} \rightarrow \mathrm{Br} \rightarrow \mathrm{I}$. According to the above listed parameters methyl- and benzyl-dibromopyridazinones proved to be the optimal precursors.

\section{Suzuki-condensation tandem reaction}

In the case when the amino compound can be produced from the azide by reduction, the Suzukicondensation tandem reaction can be chosen. Azide $\rightarrow$ amine $\rightarrow$ Suzuki reaction steps may be more efficient, than the azide $\rightarrow$ iminophosphorane $\rightarrow$ Suzuki sequence. A similar problem of selectivity was observed during preparation of $\mathbf{5 a}^{23}$ and $\mathbf{5 b}^{21}$ as mentioned above.

4-Azido-5-bromo-2-methylpyridazin-3(2H)-one could not be prepared selectively under apolar conditions (sodium azide, anhydrous toluene, 15-crown-5, heating) from 4,5-dibromo-2methylpyridazin-3(2H)-one 1 b. Ring closure of 4-nitrogen analogues $\mathbf{6 a}$ and $\mathbf{6 b}$ prepared as described above gave compounds $7 \mathbf{a}$ and $7 \mathbf{b}$. Haloamines 5 and $\mathbf{6}$ were transformed to pyridazino[4,5-c]isoquinolines 4 and 7 via Suzuki - condensation tandem reaction using 2formylphenylboronic acid. Halogen displacements by ammonia, as well as Suzuki - condensation tandem have proceeded in the case of 2-benzyl derivatives with higher yields than in the case of 2-methyl derivatives. Generally, if the amino compound can be prepared from a halogen precursor directly and selectively, then the synthesis route azide $\rightarrow$ iminophosphorane $\rightarrow$ Suzuki reaction can be neglected. In this case the target compound can be obtained from the dihalogen derivative through a haloamine and Suzuki - condensation tandem reaction with 2formylphenylboronic acid. Otherwise, Suzuki cross-coupling reaction has to be carried out with haloiminophosphoranes.

\section{Aza-Wittig - electrocyclic ring closure tandem reaction}

The above Suzuki-aza-Wittig tandem reactions started from 4,5-dibromopyridazinones. As an extension of this tandem methodology, we elaborated the synthesis of pyridazino[4,5-c]quinolines from iminophosphoranes (Scheme 2). 
<smiles>[R]n1ncc(I)cc1=O</smiles>

$8: \mathrm{R}=\mathrm{Me}$

9: $\mathrm{R}=\mathrm{Ph}$

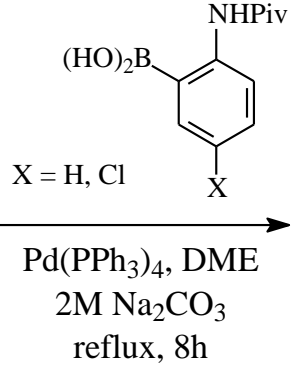

reflux, $8 \mathrm{~h}$<smiles>[X]c1ccc(NCCC)c(-c2cnn([R])c(=O)c2)c1</smiles>

10a $(64 \%)$

b $(79 \%)$

c $(75 \%)$<smiles>[X]c1ccc(N)c(-c2cnn([R])c(=O)c2)c1</smiles>

11a $(69 \%)$

b $(79 \%)$

c $(80 \%)$

1) $\mathrm{NaNO}_{2}, 37 \% \mathrm{HCl}, 0-5{ }^{\circ} \mathrm{C}, 1.5 \mathrm{~h}$

2) $\mathrm{NaN}_{3}, \mathrm{NaOAc}, 0-5^{\circ} \mathrm{C}, 1 \mathrm{~h}$

for compounds 10-15

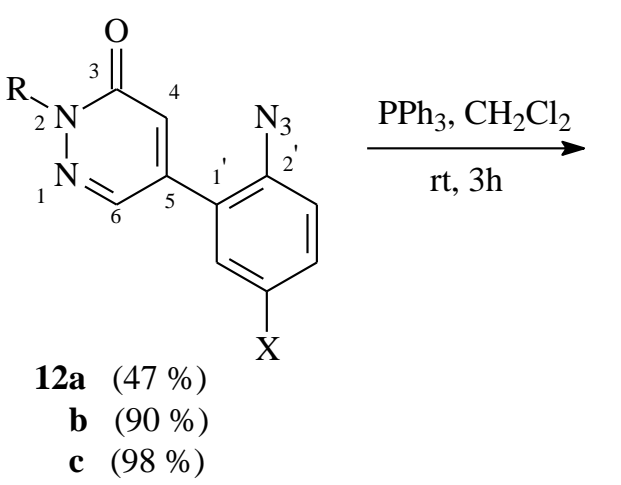<smiles>[X]c1ccc(N=[PH2+])c(-c2cnn([R])c(=O)c2)c1</smiles>

13a $(84 \%)$

b $(80 \%)$

c $(85 \%)$ a : $\mathrm{R}=\mathrm{Me}, \mathrm{X}=\mathrm{H}$

b : $\mathrm{R}=\mathrm{Me}, \mathrm{X}=\mathrm{Cl}$

c : $\mathrm{R}=\mathrm{Ph}, \mathrm{X}=\mathrm{H}$

DME: dimethoxyethane

Piv: tert-butylcarbonyl Me: methyl $\mathrm{Ph}$ : phenyl<smiles>[X]c1ccc2nc(Nc3ccccc3)c3c(=O)n([R])ncc3c2c1</smiles>

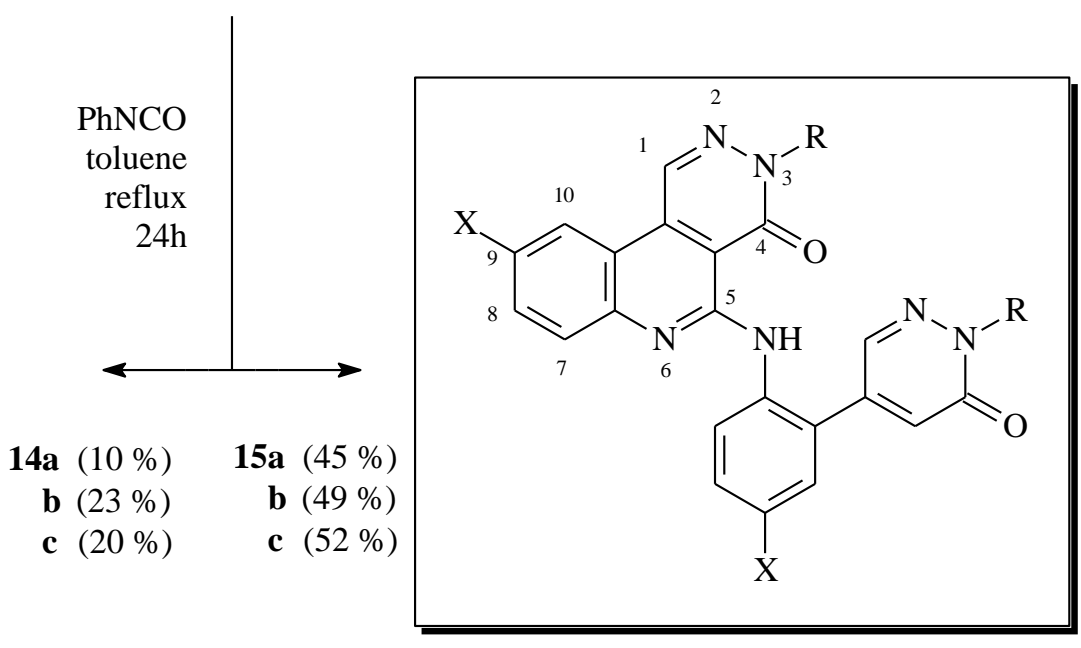

Scheme 2. Aza-Wittig - electrocyclization tandem reactions of pyridazinoiminophosphoranes. 
In an earlier paper $^{8}$ we described Suzuki cross coupling reactions of 5-iodo-2methylpyridazin-3(2H)-one $\mathbf{8}^{25}$ with arylboronic acids. This series has recently been completed using 5-iodo-2-phenylpyridazin-3(2H)-one 9. ${ }^{19}$ Accordingly, 8 and 9 were reacted with 2pivaloylamino phenylboronic acid and its 5-chloro derivative to obtain the protected aniline derivatives 10a-c. Deprotection of the protected anilines 10a-c gave the free amines 11a-c. The latter compounds were diazotized followed by treating the diazonium salts formed with $\mathrm{NaN}_{3}$ to give the corresponding azides 12a-c (Scheme 2). These azides were reacted with triphenylphosphine in dichloromethane to give iminophosphoranes 13a-c.

In the light of the reported procedure of the tandem-type reaction, ${ }^{26}$ the iminophosphoranes 13a-c were reacted with phenylisocyanate in toluene to give the corresponding carbodiimide intermediates, followed by in situ thermal cyclization at $140^{\circ} \mathrm{C}$ for $24 \mathrm{~h}$ in a one-pot reaction. The reaction product was found to be a mixture of two compounds which were easily separated by column chromatography and identified as the pyridazino[4,5-c]quinolines; 14a-c (minor products) and 15a-c (major products).

These results can be explained (see Scheme 3) on the basis of intramolecular reactions being usually favored (running faster) while the attack of another iminophosphorane at the $s p$ carbon atom of the carbodiimide led to bis-carbodiimides which underwent a ring closure reaction. Thus, the 5-phenylamino derivatives obtained via a direct intramolecular route were formed in lower yields compared to the 5-pyridazinylphenylamino compounds.

A plausible mechanism for the thermal cyclization of the carbodiimide intermediates is presented (Scheme 3). These carbodiimides, formed by aza-Wittig reaction between iminophosphoranes 13a-c and phenylisocyanate, could undergo thermal cyclization in two ways. In the case of the major product, a $N, N^{\prime}$-bis(pyridazinylphenyl)carbodiimide is formed by intermolecular reaction of the carbodiimide with another molecule of the iminophosphorane, along with elimination of phenyliminotriphenylphosphorane. In this first step the nitrogen atom of the iminophosphorane attacks at the $s p$ carbon atom of the carbodiimide as a nucleophile. Then the $N, N^{\prime}$-bis(pyridazinylphenyl)carbodiimide intermediate reacts in a subsequent intramolecular ring closure reaction giving the main product. In the case of the minor product, the carbon atom at position 4 on the pyridazinone ring initiates the nucleophile attack at the carbon of the carbodiimide and phenylamino-pyridazine is formed by a direct intramolecular step. These concurrent reaction pathways could be explained with the stronger nucleophilicity of the iminophosphorane nitrogen atom compared to the pyridazine ring carbon at position 4 . 


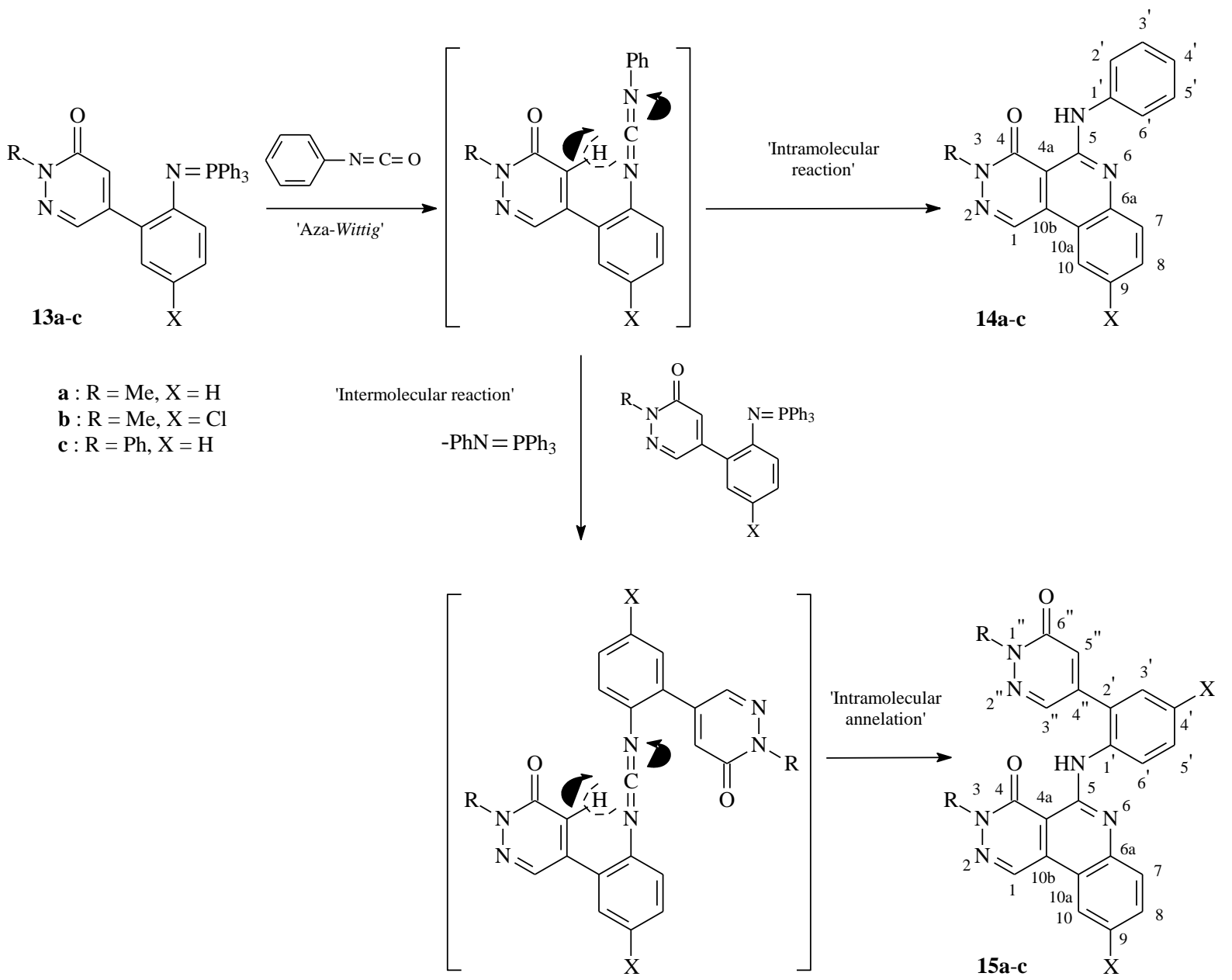

Scheme 3. Proposed mechanism of aza-Wittig - electrocyclization reactions.

Electrocyclic ring closure via a carbodiimide intermediate

i) As the aza-Wittig type reaction was successful on pyridazinones, we wanted to apply this tandem cyclization on other diazines, first on uracil derivatives. Aminophenyl uracil derivative 18 - synthesized from 5-bromo-1,3-dimethyl-uracil 16 by Suzuki cross coupling reaction via the protected amino compound $\mathbf{1 7}$ according to the method described previously ${ }^{14}$ - was the first model compound for testing the aza-Wittig type reactions (Scheme 4). 
<smiles>Cn1cc(Br)c(=O)n(C)c1=O</smiles>

16

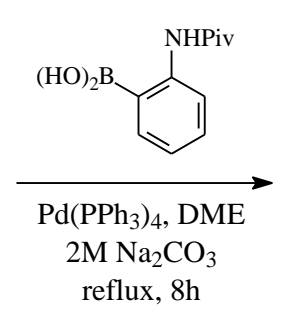

reflux, $8 \mathrm{~h}$<smiles>CCCNc1ccccc1-c1cn(C)c(=O)n(C)c1=O</smiles>

$17(91 \%)$

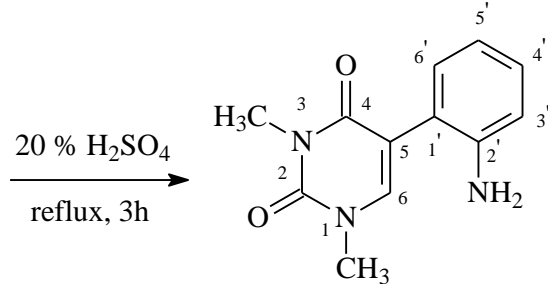

$18(88 \%)$

1) $\mathrm{NaNO}_{2}, 37 \% \mathrm{HCl}, 0-5^{\circ} \mathrm{C}, 1.5 \mathrm{~h}$

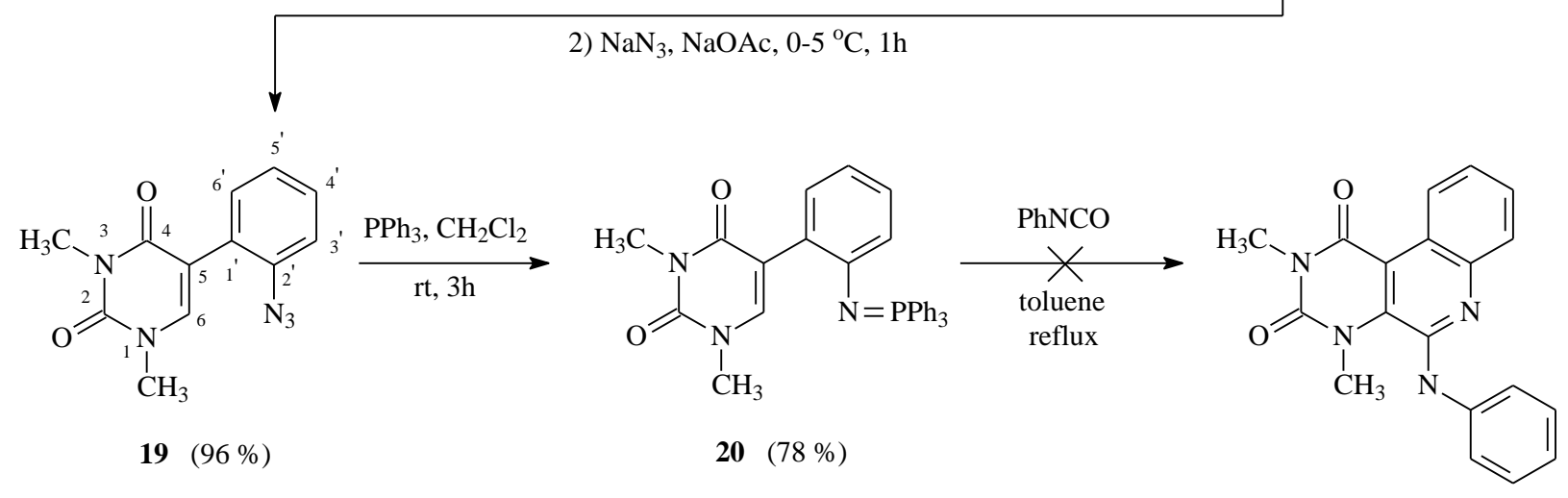

Scheme 4. Synthesis and reaction of a 5-phenyluracil derivative.

The amine 18 was first transformed to its azido-derivative 19, then in a subsequent step by reaction with triphenylphosphine to the iminiphosphorane derivative $\mathbf{2 0}$ and the latter was treated with phenylisocyanate. Our idea was to prepare a carbodiimide in situ and to carry out a subsequent cyclization. Unfortunately, the desired pyrimidoisoquinoline could not be isolated from the complex reaction mixture. This failure is probably due to the very low electron density of the uracil carbon at position 6 .

A similar situation was observed in another experiment; iminophosphorane 22 produced from azido-uracil derivative $\mathbf{2 1}^{2}$ gave with phenylisocyanate a complex mixture (Scheme 5) from which the desired pyrimidoquinoline could not be isolated. This could also be a consequence of the lower electron density of the substituted phenyl ring: the adjacent C- 6 atom of the uracil core is rather electron deficient and can withdraw electrons at ortho-position of the phenyl ring.<smiles>Cn1c(-c2ccccc2)c(N)c(=O)n(C)c1=O</smiles>

21

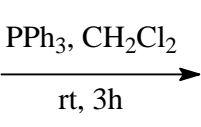<smiles>Cn1c(=O)c(N=P)c(-c2ccccc2)c(=O)n1C</smiles>

$22(82 \%)$

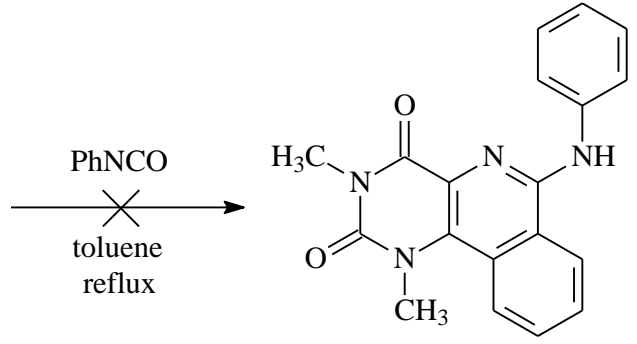

Scheme 5. Reaction of a 6-phenyluracil derivative. 
ii) Since carbodiimide was the proposed key intermediate for the tandem reactions of pyridazinones, it seemed promising to synthesize a carbodiimide directly in the case of other uracils. Suitable precursors could be found again among our own published uracils ${ }^{14}$ (Scheme 6).

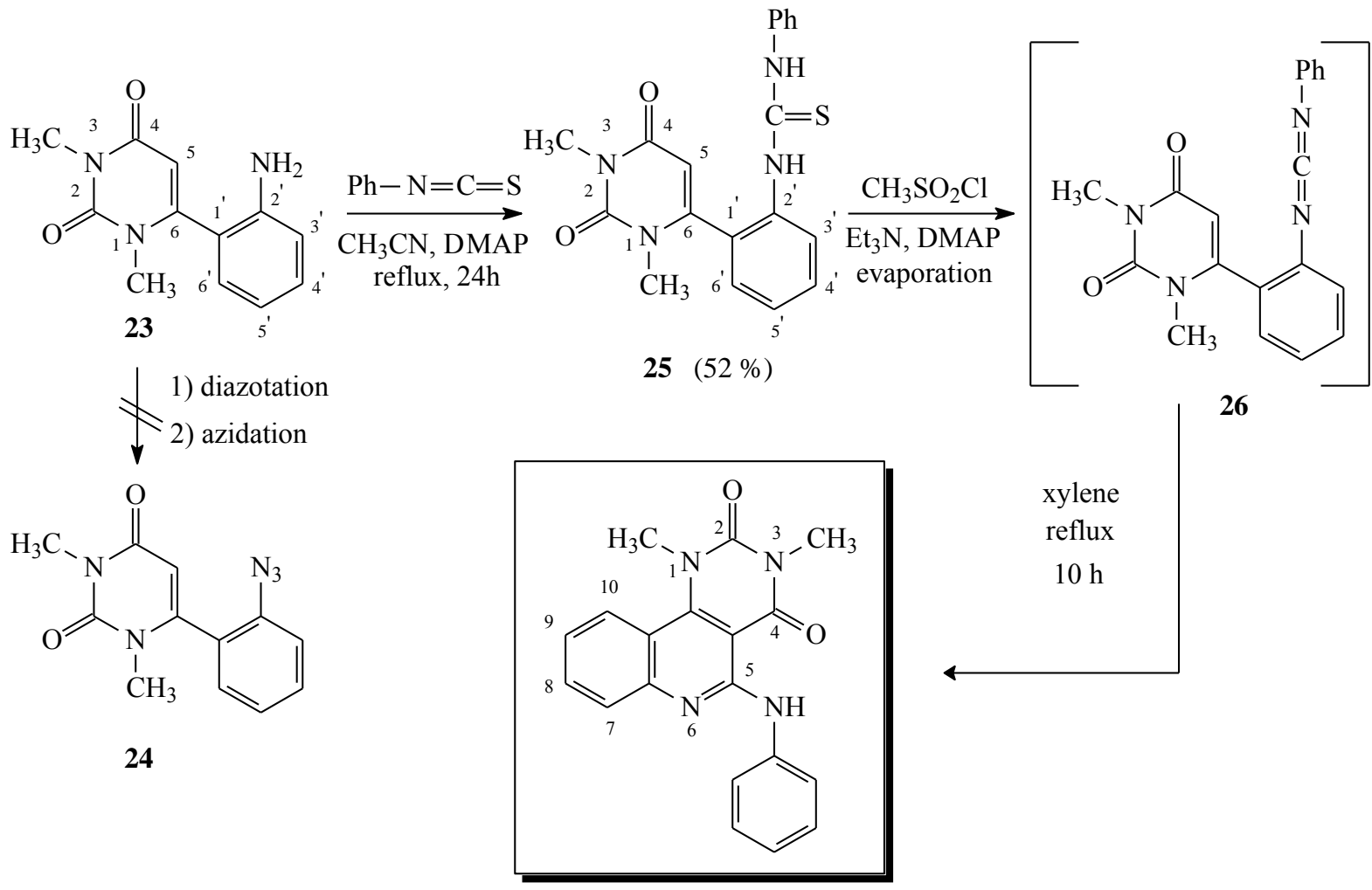

$27(66 \%)$

Scheme 6. Ring closure reaction of a 6-phenyluracil derivative.

Azido derivative $\mathbf{2 4}$ of the chosen aminophenyl uracil derivative $\mathbf{2 3}^{14}$ could not be isolated earlier; for this reason first the thiourea derivative 25 derived from 23 was prepared with phenylisothiocyanate according to a published method. ${ }^{27}$ In the next step, carbodiimide 26 was generated in situ with methanesulfonylchloride in dry toluene, then $\mathbf{2 6}$ was transformed by refluxing in xylene to the cyclized product 27. This route is not a tandem sequence, but similar to it. We observed only direct intramolecular reaction on the uracil nucleus, contrary to the analogues applied in the procedures with pyridazinones. This can be explained with the stronger nucleophilicity of the carbon atom at position 5 of the uracil core, compared to the carbodiimide nitrogen. Therefore intramolecular electrocyclization took place without formation of a dimeric carbodiimide intermediate and thus the pyrimido[5,4-c]quinoline ring system ${ }^{28}$ was directly produced instead. This finding could be explained in the following way: the nitrogen atom of the carbodiimide is a relatively weaker nucleophilic center than the carbon at position 5 of the uracil 
ring. Therefore, the direct intramolecular route is more favorable compared to the formation of a disubstituted carbodiimide, which occurred in the case of pyridazinones.

The pyrazinyl azidophenyl compound 28 (Scheme 7) prepared by us earlier ${ }^{14}$ was the next model studied in the above methodology applied for pyridazines. Similarly to the previous procedure, in the first step iminophosphorane derivative $\mathbf{2 9}$ derived from the pyrazine $\mathbf{2 8}$ was formed. Next, phenylisocyanate was reacted with this precursor but here again a complex mixture was formed from which the desired pyrazinoquinoline could not be isolated.

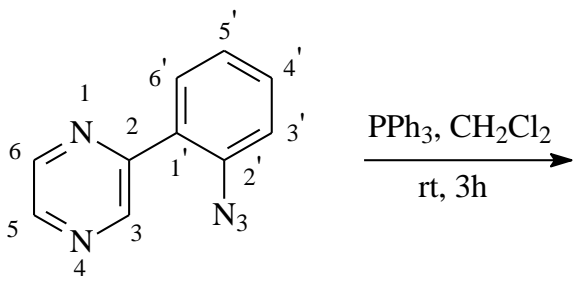

28<smiles>c1ccc(/[P+]=N\c2ccccc2-c2cnccn2)cc1</smiles>

$29(67 \%)$

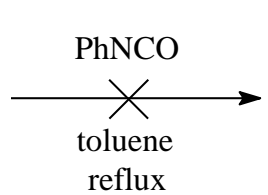

reflux<smiles>c1ccc(Nc2nc3ccccc3c3nccnc23)cc1</smiles>

Scheme 7. Reaction of a 2-phenylpiridazine derivative.

Ring closure via nitrene intermediate

According to our previous method cited above, ${ }^{8}$ a protected aniline derivative of an $N$-phenyl substituted pyridazinone (10c in Scheme 2) was also a convenient starting compound for internal ring closure following our cyclization protocol established. Thus, after deprotection of the aniline derivative to the amine this compound was diazotized to a diazonium salt which, in situ, was subjected to an aza-transfer azidation reaction to an aryl azide. Heat treatment of azide 12c generated a nitrene, attached selectively to only one of the adjacent positions of the pyridazine ring. Heating 12c in refluxing xylene gave 5H-pyrazino[4,5-b]indole 30 (Scheme 8). (The same ring closure reactions of compounds $\mathbf{1 2 \mathbf { a }}$ as well as $\mathbf{1 2 \mathbf { b }}$ were described in literature 8.)<smiles>Nc1ccccc1-c1cnn(-c2ccccc2)c(=O)c1</smiles>

12c

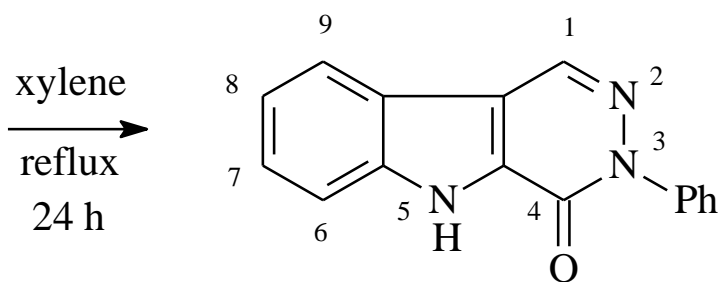

$30(59 \%)$

Scheme 8. Ring closure reaction of azidophenyl pyridazinone. 


\section{Conclusions}

Two new methods for the synthesis of the pyridazino[4,5-c]isoquinoline ring system are reported. 2-Substituted pyridazino[4,5-c]isoquinolines $\mathbf{4 a}$ and $\mathbf{4 b}$ were produced in Suzuki-azaWittig tandem reactions. Dibromo derivatives proved to be optimal halogen precursors. The pyridazinoisoquinolines $\mathbf{4 a}$ and $\mathbf{4 b}$, as well as $\mathbf{7 a}$ and $\mathbf{7 b}$ were synthesized by Suzukicondensation tandem reaction. Both the halogen displacement reactions with ammonia and Suzuki-condensation tandem reactions proceeded with higher yields when starting with one of the $N-2$ benzyl derivatives.

Application of the Suzuki-aza-Wittig tandem reaction on pyridazinones is recommended mainly in the following cases:

- if the precursor haloamine cannot be prepared regioselectively from the dihalogen compound,

- if the haloamine can be obtained only by reduction from haloazido derivative, or

- if the derivative substituted at the required position can be formed selectively in one step from the appropriate dihalogen compound.

Our present method is synthetically convenient and contains fewer steps, than the previously published route. ${ }^{5}$ Moreover, in the present method both haloamine regioisomers were produced in one step.

On the other hand, another method was developed for the synthesis of the pyridazino[4,5-c]quinoline and the pyrimido[5,4-c]quinoline ring systems. The desired compounds were produced via carbodiimide intermediates by electrocyclic ring closure.

Elaboration of these methods could open efficient accesses to fused nitrogen-containing ring systems.

\section{Experimental Section}

General. ${ }^{1} \mathrm{H}$ and ${ }^{13} \mathrm{C}$ NMR spectra were recorded on a Bruker AM $200\left({ }^{1} \mathrm{H}\right.$ NMR $200 \mathrm{MHz} ;{ }^{13} \mathrm{C}$ NMR $50 \mathrm{MHz}$ ), a Bruker Avance-500 ( ${ }^{1} \mathrm{H}$ NMR $500 \mathrm{MHz} ;{ }^{13} \mathrm{C}$ NMR $\left.125 \mathrm{MHz}\right)$, a Bruker DRX$400\left({ }^{1} \mathrm{H}\right.$ NMR $400 \mathrm{MHz} ;{ }^{13} \mathrm{C}$ NMR $\left.100 \mathrm{MHz}\right)$ or a Varian MERCURY plus $\left({ }^{1} \mathrm{H}\right.$ NMR $400 \mathrm{MHz}$; ${ }^{13} \mathrm{C}$ NMR $100 \mathrm{MHz}$ ) spectrometer in the solvent indicated at room temperature, using the ${ }^{2} \mathrm{H}$ signal of the solvent as the lock. The ${ }^{1} \mathrm{H}$ and ${ }^{13} \mathrm{C}$ chemical shifts were referred to TMS $\left(\delta_{\text {TMS }}=0\right.$ ppm). Chemical shifts $(\delta)$ are given in ppm and coupling constant $(J)$ values in $\mathrm{Hz}$. The structures of compounds were elucidated using COSY, HSQC, HMBC (NMR) methods. Melting points were determined in a Büchi Melting Point B-540 apparatus and the values are uncorrected. IR spectra were recorded in potassium bromide pellets with a Perkin-Elmer 1600 FT-IR spectrophotometer. Mass spectra were taken on a Finnigan MAT 8430 spectrometer [resolution: 1250, ion accelerating voltage: $3 \mathrm{kV}$, ion source temperature: $250{ }^{\circ} \mathrm{C} / \mathrm{Electron}$ Ionization Mass Spectra (EIMS) and Chemical Ionization Mass Spectra (CIMS), $25{ }^{\circ} \mathrm{C} / \mathrm{Fast}$ 
Atom Bombardment Mass Spectra (FABMS)] and a quadrupole-time-of-flight mass spectrometer (Q-Tof-II, Micromass, Manchester, UK; cone voltage approx. 35V and capillary voltage approx. $3.3 \mathrm{kV}$ ). Elemental analyses were performed on a Carlo Erba Elemental Analyzer Model 1012 apparatus.

Commercially available solvents were purified by standard procedures prior to use, whereas reagents (Reanal, Budapest, or Sigma-Aldrich Kft., Budapest) were used as received. Standard flash chromatography on silica (Kieselgel 60, Aldrich, 0.040-0.063 mm) and/or recrystallization as indicated, was applied for purification of crude products. Thin layer chromatography was done on commercially available silica plates (Silica gel $60 \mathrm{~F}_{254}$, Merck).

Compounds $1 \mathbf{a},{ }^{22} \mathbf{1 b},{ }^{21} \mathbf{1 c},{ }^{17} \mathbf{5 b},{ }^{21} \mathbf{6 b},{ }^{21} \mathbf{8},{ }^{25} \mathbf{9},{ }^{19} \mathbf{1 0 a},{ }^{8} \mathbf{1 0 b},{ }^{8} \mathbf{1 1 a},{ }^{8} \mathbf{1 1 b},{ }^{8} \mathbf{1 2 a},{ }^{8} \mathbf{1 2 b},{ }^{8} \mathbf{2 1},{ }^{2,14} \mathbf{2 3}^{14}$ and $\mathbf{2 8}^{14}$ were synthesized according to the literature. Compound 5a was also synthesized according to the literature ${ }^{23}$ and $\mathbf{6 a}$ was also isolated from the same reaction mixture. Spectroscopic data of $\mathbf{1 d},{ }^{20} \mathbf{2 b},{ }^{24} \mathbf{2} \mathbf{c},{ }^{24} \mathbf{4 a},{ }^{5} \mathbf{4 b},{ }^{5} \mathbf{7 a}^{5}$ and $\mathbf{7} \mathbf{b}^{5}$ are corresponding to those reported in the literature for identical compounds produced via routes different from the ones described herein.

\section{Method A. General procedure for the reaction of 1a-d dihalopyridazinones with sodium azide}

To a suspension of the 4,5-dihalopyridazinone derivative 1a-d $(8.3 \mathrm{mmol})$ in ethanol $(58 \mathrm{~mL})$ and water $(29 \mathrm{~mL})$, sodium azide $(16.6 \mathrm{mmol})$ was added, and the mixture was refluxed until the starting material was consumed as judged by TLC analysis. The hot reaction mixture was filtered and the filtrate was evaporated to dryness in vacuo. The solid residue was taken up in water (20 $\mathrm{mL})$ and extracted with chloroform $(3 \times 20 \mathrm{~mL})$. The combined organic layers were dried over anhydrous magnesium sulfate. The solvent was evaporated in vacuo, and the crude product 2a-d was purified by column chromatography or by recrystallization as given below.

5-Azido-2-benzyl-4-bromopyridazin-3(2H)-one (2a). Reaction time: $4 \mathrm{~h}$; eluent used for flash column chromatography: toluene. Yellow crystals, yield $1.33 \mathrm{~g}, 52 \%, \mathrm{mp} 66-67{ }^{\circ} \mathrm{C}$; $R_{\mathrm{f}}$ (toluenemethanol 4:1): 0.69. IR ( $\left.v_{\max }, \mathrm{cm}^{-1}\right): 3288,3104,2960,2132,1638,1594,1420,1354,1314$, 1218, 1112, 1074, 742, $700 \mathrm{~cm}^{-1} .{ }^{1} \mathrm{H}$ NMR (200 MHz, $\left.\mathrm{CDCl}_{3}\right): \delta \mathrm{H} 7.62(1 \mathrm{H}, \mathrm{s}, \mathrm{H}-6), 7.46-7.26$ (m, 5H, phenyl protons), $5.32\left(\mathrm{~s}, 2 \mathrm{H}, \mathrm{CH}_{2}\right) .{ }^{13} \mathrm{C} \mathrm{NMR}\left(50 \mathrm{MHz}, \mathrm{CDCl}_{3}\right): \delta \mathrm{c} 157.3(\mathrm{C}-3), 141.7$ (C-5), 135.3 (C-1'), 129.0 (C-2',6'), 128.8 (C-6), 128.6 (C-3',5'), 128.3 (C-4'), 113.6 (C-4), 56.4 $\left(\mathrm{CH}_{2}\right)$; C' are benzyl aromatic carbons. Anal. Calcd for $\mathrm{C}_{11} \mathrm{H}_{8} \mathrm{BrN}_{5} \mathrm{O}$ (306.12): C, 43.16; H, 2.63; N, 22.88\%. Found: C, 43.13; H, 2.46; N, 22.63\%.

5-Azido-4-bromo-2-methylpyridazin-3(2H)-one (2b). Reaction time: 9 h. Yellow crystals, yield 53\%, $1.01 \mathrm{~g}$, mp $104-106{ }^{\circ} \mathrm{C}$ (from dry ethanol, lit. ${ }^{24} \mathrm{mp} 103-104{ }^{\circ} \mathrm{C}$ ).

5-Azido-4-chloro-2-methylpyridazin-3(2H)-one (2c). Reaction time: $32 \mathrm{~h}$; eluent used for flash column chromatography: toluene. Yellow crystals, yield $31 \%, 0.47 \mathrm{~g}, \mathrm{mp} 86-86.5{ }^{\circ} \mathrm{C}$ (lit. ${ }^{24} \mathrm{mp}$ 90-91 $\left.{ }^{\circ} \mathrm{C}\right)$. 
5-Azido-4-iodo-2-methylpyridazin-3(2H)-one (2d). Reaction time: $12 \mathrm{~h}$. Yellow crystals, yield $73 \%, 1.68 \mathrm{~g}, \mathrm{mp} 116-118{ }^{\circ} \mathrm{C}$ (from dichloromethane); $R_{\mathrm{f}}$ (toluene-methanol 4:1): 0.49. IR ( $v_{\max }$, $\left.\mathrm{cm}^{-1}\right): 3032,2940,2126,1624,1580,1546,1382,1318,1292,688 .{ }^{1} \mathrm{H}$ NMR (200 MHz, $\left.\mathrm{CD}_{3} \mathrm{OD}\right): \delta \mathrm{H} 7.81$ (s, 1H, H-6), $3.80\left(\mathrm{~s}, 3 \mathrm{H}, \mathrm{CH}_{3}\right) .{ }^{13} \mathrm{C} \mathrm{NMR}\left(50 \mathrm{MHz}, \mathrm{CD}_{3} \mathrm{OD}\right): \delta \mathrm{c} 158.2(\mathrm{C}-3)$, 149.4 (C-5), 130.2 (C-6), $91.6(\mathrm{C}-4), 41.6\left(\mathrm{CH}_{3}\right)$. Anal. Calcd for $\mathrm{C}_{5} \mathrm{H}_{4} \mathrm{IN}_{5} \mathrm{O}$ (277.03): C, 21.68; H, 1.46; N, 25.28\%. Found: $C, 21.58 ; \mathrm{H}, 1.33 ; \mathrm{N}, 24.81 \%$.

\section{Method B. General procedure for the reaction of azides with triphenylphosphine}

A round-bottom flask was purged with argon and charged with the appropriate azido compound derivative $(13.10 \mathrm{mmol})$ and dry dichloromethane $(100 \mathrm{~mL})$. Under stirring, the mixture was flushed with argon for approximately $5 \mathrm{~min}$. Subsequently triphenylphosphine (13.50 mmol) was added and the reaction mixture was stirred at room temperature for three hours. The solvent was evaporated in vacuo, and the crude solid product 3a-d was recrystallized from dichloromethane or purified by column chromatography.

2-Benzyl-4-bromo-5-(triphenylphosphoranylideneamino)pyridazin-3(2H)-one

(3a). Colourless crystals, yield $81 \%, 5.70 \mathrm{~g}, \mathrm{mp} 197-198{ }^{\circ} \mathrm{C} ; R_{\mathrm{f}}$ (chloroform-ethyl acetate 9:1): 0.76. IR $\left(v_{\max }, \mathrm{cm}^{-1}\right): 3050,2924,1624,1568,1476,1412,1354,1326,1198,1108,720,692,528 .{ }^{1} \mathrm{H}$ NMR (200 MHz, $\mathrm{CDCl}_{3}$ ): $\delta \mathrm{H}$ 7.83-7.18 (m, 20H, phenyl protons), 6.96 (s, 1H, H-6), 5.23 (s, 2H, $\left.\mathrm{CH}_{2}\right) .{ }^{13} \mathrm{C}$ NMR $\left(50 \mathrm{MHz}, \mathrm{CDCl}_{3}\right)$ : $\delta \mathrm{C} 159.9$ (C-3), $151.2(\mathrm{C}-5), 137.0$ (C-1'), $133.8(\mathrm{C}-6), 132.7$ (C-4”), 132.4 (C-2”,6”), 129.2 (C-1”), 129.1 (C-3”,5”), 128.8 (C-2',6'), 128.4 (C-3',5'), 127.5 (C-4'), 109.9 (C-4), $54.9\left(\mathrm{CH}_{2}\right)$; C' are benzyl aromatic carbons, C' are phenyl carbons; P-C coupling constants: ${ }^{1} J_{(\mathrm{P}, \mathrm{C}-1 ")} 101,{ }^{2} \boldsymbol{J}_{(\mathrm{P}, \mathrm{C}-2 ",-6 ")} 10,{ }^{3} J_{(\mathrm{P}, \mathrm{C}-3 ",-5 ")} 12,{ }^{4} J_{(\mathrm{P}, \mathrm{C}-4 ")} 2.2,{ }^{3} J_{(\mathrm{P}, \mathrm{C}-4)} 24$, and ${ }^{3} J_{(\mathrm{P}, \mathrm{C}-6)} 11 \mathrm{~Hz}$. Anal. Calcd for $\mathrm{C}_{29} \mathrm{H}_{23} \mathrm{BrN}_{3} \mathrm{OP}$ (540.381): C, 64.46; H, 4.29; N, 7.78\%. Found: C, 64.54; H, 4.19; N, 7.71\%.

\section{4-Bromo-2-methyl-5-(triphenylphosphoranylideneamino)pyridazin-3(2H)-one}

(3b). Colourless crystals, yield $72 \%, 4.39 \mathrm{~g}, \mathrm{mp} 223-223.8^{\circ} \mathrm{C} ; R_{\mathrm{f}}$ (toluene-methanol 7:3): 0.33. IR $\left(v_{\max }, \mathrm{cm}^{-1}\right): 3052,2940,1638,1568,1478,1436,1396,1332,1226,1108,942,720,692,530$, 504. ${ }^{1} \mathrm{H}$ NMR (500 MHz, DMSO-d6): $\delta$ н 7.82-7.63 (m, 15H, phenyl protons), 6.88 (s, 1H, H-6), 3.50 (s, 3H, $\left.\mathrm{CH}_{3}\right) .{ }^{13} \mathrm{C}$ NMR (125 MHz, DMSO-d6): $\delta \mathrm{c} 158.3$ (C-3), 150.9 (C-5), 132.9 (C-4'), 132.3 (C-6), 132.1 (C-2',6'), 129.4 (C-3',5'), 128.4 (C-1'), 110.0 (C-4), $39.4\left(\mathrm{CH}_{3}\right)$; C' are phenyl carbons; P-C coupling constants: ${ }^{1} J_{\left(\mathrm{P}, \mathrm{C}-1^{\prime}\right)} \quad 101,{ }^{2} J_{\left(\mathrm{P}, \mathrm{C}-2^{\prime},-6^{\prime}\right)} 11,{ }^{3} J_{\left(\mathrm{P}, \mathrm{C}-3^{\prime},-5^{\prime}\right)} 12,{ }^{4} J_{\left(\mathrm{P}, \mathrm{C}-4^{\prime}\right)}$ 2.3, ${ }^{3} J_{(\mathrm{P}, \mathrm{C}-4)} 24$, and ${ }^{3} J_{(\mathrm{P}, \mathrm{C}-6)} 12 \mathrm{~Hz}$. Anal. Calcd for $\mathrm{C}_{23} \mathrm{H}_{19} \mathrm{BrN}_{3} \mathrm{OP}$ (464.30): C, 59.50; H, 4.12; N, 9.05\%. Found: C, 59.95; H, 4.07; N, 9.06\%.

4-Chloro-2-methyl-5-(triphenylphosphoranylideneamino)pyridazin-3(2H)-one

(3c). Colourless crystals, yield 56\%, $3.09 \mathrm{~g}, \mathrm{mp} 218-219^{\circ} \mathrm{C} ; R_{\mathrm{f}}$ (toluene-methanol 4:1): 0.45. IR ( $v_{\max }$, $\left.\mathrm{cm}^{-1}\right): 3446,3048,2928,2856,1630,1570,1478,1394,1326,1106,720,688,528 .{ }^{1} \mathrm{H}$ NMR (200 MHz, DMSO-d6): $\delta \mathrm{H}$ 7.85-7.58 (m, 15H, phenyl protons), 6.99 (s, 1H, H-6), 3.50 (s, 3H, $\left.\mathrm{CH}_{3}\right) .{ }^{13} \mathrm{C}$ NMR (50 MHz, DMSO-d6): $\delta \mathrm{c} 158.0$ (C-3), 149.0 (C-5), 133.2 (C-6), 132.9 (C-4'), 132.1 (C-2',6'), 129.4 (C-3',5'), 128.7 (C-4), 128.6 (C-1'), $39.2\left(\mathrm{CH}_{3}\right)$. C' are phenyl carbons; PC coupling constants: ${ }^{1} J\left(\mathrm{P}, \mathrm{C}-1^{\prime}\right) \quad 101,{ }^{2} J\left(\mathrm{P}, \mathrm{C}-2^{\prime}, 6^{\prime}\right) \quad 10,{ }^{3} J\left(\mathrm{P}, \mathrm{C}-3^{\prime}, 5^{\prime}\right) \quad 12,{ }^{4} J\left(\mathrm{P}, \mathrm{C}-4^{\prime}\right) \quad 2.2,{ }^{3} J(\mathrm{P}$, 
C-4) 24, and ${ }^{3} J(\mathrm{P}, \mathrm{C}-6) 13 \mathrm{~Hz}$. Anal. Calcd for $\mathrm{C}_{23} \mathrm{H}_{19} \mathrm{ClN}_{3} \mathrm{OP}$ (419.85): C, 65.80; H, 4.56; N, 10.01\%. Found: C, 65.69; H, 4.46; N, 9.89\%.

4-Iodo-2-methyl-5-(triphenylphosphoranylideneamino)pyridazin-3(2H)-one (3d). Yellow crystals, yield $31 \%, 2.07 \mathrm{~g}, \mathrm{mp} 234-235{ }^{\circ} \mathrm{C} ; R_{\mathrm{f}}$ (toluene-methanol 4:1): 0.41. IR $\left(v_{\max }, \mathrm{cm}^{-1}\right)$ : 3050, 2936, 1626, 1566, 1478, 1430, 1398, 1334, 1308, 1106, 950, 720, 690, 528, 508. ${ }^{1} \mathrm{H}$ NMR (200 MHz, $\left.\mathrm{CDCl}_{3}\right): \delta \mathrm{H} 7.85-7.40\left(\mathrm{~m}, 15 \mathrm{H}\right.$, phenyl protons), $6.77(\mathrm{~s}, 1 \mathrm{H}, \mathrm{H}-6), 3.68\left(\mathrm{~s}, 3 \mathrm{H}, \mathrm{CH}_{3}\right)$. ${ }^{13} \mathrm{C}$ NMR (50 MHz, $\mathrm{CDCl}_{3}$ ): $\delta \mathrm{c} 155.5$ (C-3), 149.5 (C-5), 132.6 (C-4'), 132.4 (C-2',6'), 131.6 (C-6), 129.0 (C-3',5'), 128.9 (C-1'), $92.9(\mathrm{C}-4), 40.1\left(\mathrm{CH}_{3}\right)$; C' are phenyl carbons; P-C coupling constants: ${ }^{1} J_{\left(\mathrm{P}, \mathrm{C}-1^{\prime}\right)} \quad 101,{ }^{2} J_{\left(\mathrm{P}, \mathrm{C}-2^{\prime},-6^{\prime}\right)} 10,{ }^{3} J_{\left(\mathrm{P}, \mathrm{C}-3^{\prime},-5^{\prime}\right)} 12,{ }^{4} J_{\left(\mathrm{P}, \mathrm{C}-4^{\prime}\right)} 2.6,{ }^{3} J_{(\mathrm{P}, \mathrm{C}-4)} 23$, and ${ }^{3} J_{(\mathrm{P}, \mathrm{C}-6)} 11$ Hz. Anal. Calcd for $\mathrm{C}_{23} \mathrm{H}_{19} \mathrm{IN}_{3} \mathrm{OP}$ (511.29): C, 54.03; H, 3.75; N, 8.22\%. Found: C, 54.01; H, $3.62 ; \mathrm{N}, 8.11 \%$.

Method C. General procedure for the synthesis of (4a,b) via Suzuki-reaction from the appropriate haloiminophosphoranes

A round-bottom flask was purged with argon and charged with the iminophosphorane derivative 3a-d $(2.0 \mathrm{mmol})$ and dry 1,2-dimethoxyethane $(30 \mathrm{~mL})$. While stirring, the mixture was flushed with argon for approximately $10 \mathrm{~min}$. Subsequently tetrakis(triphenylphosphine)-palladium $(0)$ $(0.12 \mathrm{mmol}), 2$-formylphenylboronic acid $(2.8 \mathrm{mmol})$ and sodium carbonate solution $(2 \mathrm{M}, 10$ $\mathrm{mL}$ ) were added and the reaction mixture was heated at the temperature given below. The solvent was evaporated in vacuo, the crude black oil obtained was taken up in water (80 $\mathrm{mL})$ and extracted with chloroform $(3 \times 80 \mathrm{~mL})$. The combined organic layers were dried over anhydrous magnesium sulfate. The solvent was evaporated in vacuo, and the brown residue was purified by flash column chromatography as given below.

2-Benzyl-pyridazino[4,5-c]isoquinolin-1(2H)-one (4a). Starting from 2-benzyl-4-bromo-5(triphenyliminophosphoranyl)pyridazin-3(2H)-one 3a, reaction conditions: $7 \mathrm{~h}$ at $120{ }^{\circ} \mathrm{C}$; eluent used for flash column chromatography: toluene-methanol 95:5. Colourless crystals, yield 92\%, $0.39 \mathrm{~g}, \mathrm{mp} 173-174{ }^{\circ} \mathrm{C}$ (lit. ${ }^{5} \mathrm{mp} 173{ }^{\circ} \mathrm{C}$ ).

2-Methyl-pyridazino[4,5-c]isoquinolin-1(2H)-one (4b). (a) Starting from 4-bromo-2-methyl-5(triphenyliminophosphoranyl)pyridazin-3(2H)-one $\mathbf{3 b}$, reaction conditions: $7 \mathrm{~h}$ at $120^{\circ} \mathrm{C}$; eluent used for flash column chromatography: toluene-methanol 4:1. Colourless crystals, yield 75\%, $0.31 \mathrm{~g}$, mp $155.5-156{ }^{\circ} \mathrm{C}$ (lit. ${ }^{5} \mathrm{mp} 149-151{ }^{\circ} \mathrm{C}$ ). (b) Starting from 4-chloro-2-methyl-5(triphenyliminophosphoranyl)pyridazin-3(2H)-one 3c, reaction conditions: $140 \mathrm{~h}$ at $150^{\circ} \mathrm{C}$; eluent used for flash column chromatography: toluene-methanol 99:1. yield 6\%, $0.03 \mathrm{~g}$, mp 154 ${ }^{\circ} \mathrm{C}$. (c) Starting from 4-iodo-2-methyl-5-(triphenyliminophosphoranyl)pyridazin-3(2H)-one 3d, reaction conditions: $18 \mathrm{~h}$ at $120{ }^{\circ} \mathrm{C}$; eluent used for flash column chromatography: toluenemethanol 9:1. yield 64\%, $0.27 \mathrm{~g}, \mathrm{mp} 155^{\circ} \mathrm{C}$. 
Method D. General procedure for the synthesis of $(4 a, b)$, via Suzuki-reaction from the appropriate haloamines

A round-bottom flask was purged with argon and charged with the amino derivative $\mathbf{5 a}$ or $\mathbf{5 b}$ $(2.0 \mathrm{mmol})$ and dry 1,2-dimethoxyethane $(30 \mathrm{~mL})$. While stirring, the mixture was flushed with argon for approximately $10 \mathrm{~min}$. Subsequently tetrakis(triphenylphosphine)-palladium(0) (0.12 $\mathrm{mmol})$, 2-formylphenylboronic acid $(4.0 \mathrm{mmol})$ and sodium carbonate solution $(2 \mathrm{M}, 2.0 \mathrm{~mL})$ were added, and the reaction mixture was heated at $110{ }^{\circ} \mathrm{C}$ for 12 hours. The reaction mixture was poured onto ice-water $(60 \mathrm{~mL})$, was extracted with dichloromethane $(3 \times 45 \mathrm{~mL})$ and the combined organic layers were dried over anhydrous sodium sulfate. The solvent was evaporated in vacuo and the residue was purified by flash column chromatography as given below.

2-Benzyl-pyridazino[4,5-c]isoquinolin-1(2H)-one (4a). Starting from 5-amino-2-benzyl-4bromopyridazin-3(2H)-one 5a, eluent used for flash column chromatography: toluene-methanol 4:1. Yield $54 \%, 0.31 \mathrm{~g}, \mathrm{mp} 171-172{ }^{\circ} \mathrm{C}\left(\text { lit.: } 173^{\circ} \mathrm{C}\right)^{5}$.

2-Methyl-pyridazino[4,5-c]isoquinolin-1(2H)-one (4b). Starting from $\mathbf{5 b}$, eluent used for flash column chromatography: toluene-methanol 7:3. Yield , $62 \%, 0.39 \mathrm{~g}$, mp 154-155 ${ }^{\circ} \mathrm{C}$ (lit.: 149$\left.151^{\circ} \mathrm{C}\right)^{5}$.

Method E. Synthesis and characterization of haloamines (5a) and (6a)

5-Amino-2-benzyl-4-bromopyridazin-3(2H)-one (5a). Reaction of 2-benzyl-4,5dibromopyridazin-3(2H)-one 1a $(7.12 \mathrm{mmol})$ with ammonia $(50 \mathrm{~mL}, 25 \%)$ was run in an autoclave $\left(160^{\circ} \mathrm{C}, 6 \mathrm{~h}\right)$ under stirring. After cooling, the resulting precipitate was collected by filtration, washed with water, and recrystallized to give 5a as Colourless crystals, yield 55\%, $1.10 \mathrm{~g}$, mp 230.5-231 ${ }^{\circ} \mathrm{C}$ (from ethanol, lit. ${ }^{24} \mathrm{mp} 217-219{ }^{\circ} \mathrm{C}$ ); $R_{\mathrm{f}}$ (toluene-methanol 20:1): 0.10. IR $\left(v_{\max }, \mathrm{cm}^{-1}\right): 3444,3288,3240,3164,3026,2960,1636,1600,1454,1418,1206,738,700 .{ }^{1} \mathrm{H}$ NMR (500 MHz, DMSO-d6): $\delta \mathrm{H} 7.55$ (s, 1H, H-6), 7.31-7.24 (m, 5H, phenyl protons), 6.76 (s, $\left.2 \mathrm{H}, \mathrm{NH}_{2}\right), 5.16\left(\mathrm{~s}, 2 \mathrm{H}, \mathrm{CH}_{2}\right) .{ }^{13} \mathrm{C}$ NMR (125 MHz, DMSO-d6): $\delta \mathrm{c} 157.1$ (C-3), $147.4(\mathrm{C}-5)$, 137.3 (C-1'), 129.6 (C-6), 128.4 (C-3',5'), 127.6 (C-2',6'), 127.3 (C-4'), 95.4 (C-4), $54.0\left(\right.$ CH $\left._{2}\right)$; $\mathrm{C}^{\prime}$ are phenyl carbons. Anal. Calcd for $\mathrm{C}_{11} \mathrm{H}_{10} \mathrm{BrN}_{3} \mathrm{O}$ (280.12): C, 47.17; H, 3.60; N, 15.00; Br, 28.52\%. Found: C, 47.08; H, 3.37; N, 14.96; Br, 28.70\%.

4-Amino-2-benzyl-5-bromopyridazin-3(2H)-one (6a). Obtained by flash chromatography purification of the mother liquor of 5a (prepared from 1a). Pale yellow crystals, yield 11\%, 0.22 g, mp 109.7-110.2 ${ }^{\circ} \mathrm{C} ; R_{\mathrm{f}}$ (toluene-methanol 20:1): 0.37. IR $\left(v_{\max }, \mathrm{cm}^{-1}\right): 3470,3384,3300,2962$, 2928, 1604, 1542, 1510, 1454, 1336, 1224, 878, 836. ${ }^{1} \mathrm{H}$ NMR (500 MHz, DMSO-d6): $\delta_{\text {H }} 7.79$ (s, 1H, H-6), 7.32-7.26 (m, 5H, phenyl protons), $6.72\left(\mathrm{~s}, 2 \mathrm{H}, \mathrm{NH}_{2}\right), 5.20\left(\mathrm{~s}, 2 \mathrm{H}, \mathrm{CH}_{2}\right) .{ }^{13} \mathrm{C} \mathrm{NMR}$ (125 MHz, DMSO-d6): $\delta$ c 154.2 (C-3), 143.1 (C-4), 139.4 (C-6), 136.7 (C-1'), 128.4 (C-3',5'), 127.8 (C-2',6'), 127.4 (C-4'), 95.5 (C-5), $54.1\left(\mathrm{CH}_{2}\right)$; C' are phenyl carbons; MS (ESI): $m / z(\%)$ 92, 201, 203, 280, 282; HRMS (ESI) calcd. for $\mathrm{C}_{11} \mathrm{H}_{10} \mathrm{BrN}_{3} \mathrm{O}[\mathrm{M}+\mathrm{H}]^{+}$: 280.0085, found: 280.0078 .

Compounds 7a,b were prepared according to Method D above. 
3-Benzyl-piridazino[4,5-c]isoquinolin-4(3H)-one (7a). Starting from 4-amino-2-benzyl-5bromopyridazin-3(2H)-one $\mathbf{6 a}$, eluent used for flash column chromatography: toluene-methanol 4:1. Yield 70\%, $0.08 \mathrm{~g}, \mathrm{mp} 239-240{ }^{\circ} \mathrm{C}$ (lit.: $\left.240{ }^{\circ} \mathrm{C}\right)^{5}$.

3-Methylpiridazino[4,5-c]isoquinolin-4(3H)-one (7b). Starting from 4-amino-5-bromo-2methylpyridazin-3 $2 H$ )-one $\mathbf{6 b}$, eluent used for flash column chromatography: toluene-methanol 7:3. Yield 59\%, 0.12 g, mp 287-290 ${ }^{\circ} \mathrm{C}$ (lit.: 287-295 ${ }^{\circ} \mathrm{C}$ ) ${ }^{5}$.

$N$-[2-(6-Oxo-1-phenyl-1,6-dihydropyridazin-4-yl)phenyl]pivalamide (10c). 5-Iodo-2-phenyl3(2H)-pyridazinone 9 (2.98 g, $10.00 \mathrm{mmol})$ and tetrakis(triphenylphosphine)-palladium(0) (0.58 $\mathrm{g}, 0.50 \mathrm{mmol})$ as a catalyst were dissolved in dimethoxyethane $\left(60 \mathrm{~mL}\right.$, distilled over $\left.\mathrm{SnCl}_{2}\right)$ and were stirred under argon at room temperature for $30 \mathrm{~min}$. Pivaloylamino-phenylboronic acid $(2.76 \mathrm{~g}, 12.50 \mathrm{mmol})$ and sodium carbonate solution $(2 \mathrm{M}, 10 \mathrm{~mL})$ were then added and the mixture was refluxed for 8 hours. The reaction mixture was poured onto ice-water $(80 \mathrm{~mL})$ and was extracted with chloroform $(3 \times 100 \mathrm{~mL})$. Evaporation of the organic layer gave a crude product which was recrystallized. Yellow crystals, yield 75\%, $2.61 \mathrm{~g}, \mathrm{mp} 191.1-192.0{ }^{\circ} \mathrm{C}$ (from acetonitrile); $R_{\mathrm{f}}$ (toluene-metanol 4:1): 0.25. IR ( $\left.v_{\max }, \mathrm{cm}^{-1}\right): 3308,2956,1660,1512,1450,1298$, 758, 694. ${ }^{1} \mathrm{H}$ NMR (500 MHz, $\mathrm{CDCl}_{3}$ ): $\delta \mathrm{H} 7.94$ (d, J3',5, $2.1 \mathrm{~Hz}, 1 \mathrm{H}, \mathrm{H}-3$ ') 7.77 (d, J5,6 8.1 Hz, 1H, H-6), 7.60 (d, J2",3"=J", 6" 7.7 Hz, 2H, H-2",6”), 7.58 (s, 1H, NH), 7.48 (m, 1H, H-5), 7.45 (t, 2H, H-3",5”), 7.38 (t, J3",4"=J4",5" $8.0 \mathrm{~Hz}, 1 \mathrm{H}, \mathrm{H}-4$ "), 7.33 (m, 1H, H-3), 7.32 (m, 1H, H-4), 6.96

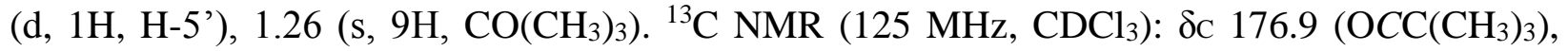
159.6 (C-6'), 143.1 (C-4'), 141.0 (C-1’), 137.9 (C-3’), 134.7 (C-1), 130.7 (C-5), 129.2 (C-3), 128.7 (C-3",5”), 128.5 (C-5'), 128.4 (C-2), 128.3 (C-4”), 126.2 (C-4), 125.8 (C-6), 125.1 (C-

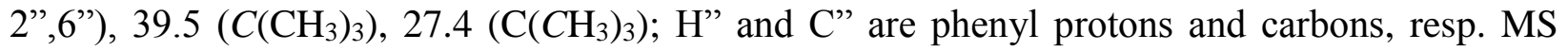
(ESI): $m / z$ (\%) 310, 327, 344, 365. HRMS (ESI) calcd. for $\mathrm{C}_{21} \mathrm{H}_{21} \mathrm{~N}_{3} \mathrm{O}_{2}[\mathrm{M}+\mathrm{H}]^{+}: 348.1712$, found: 348.1705 .

5-(2-Aminophenyl)-2-phenylpyridazin-3(2H)-one (11c). $\mathrm{N}$-[2-(6-Oxo-1-phenyl-1,6-dihydropyridazin-4-yl)phenyl]pivalamide 10c $(2.29 \mathrm{~g}, 6.59 \mathrm{mmol})$ was added to sulfuric acid $(20 \%, 115$ $\mathrm{mL}$ ) and the mixture was refluxed for 3 hours. After cooling, the $\mathrm{pH}$ of the mixture was adjusted to $\mathrm{pH} 8$ by addition of aqueous ammonia (25\%). The mixture was then extracted with chloroform $(3 \times 100 \mathrm{~mL})$ and the crude product obtained after evaporation of the organic layer was recrystallized. Pale brown crystals, yield $80 \%, 1.38 \mathrm{~g}, \mathrm{mp} 144.5-145.8{ }^{\circ} \mathrm{C}$ (from acetonitrile); $R_{\mathrm{f}}$ (chloroform-methanol 40:1): 0.38. IR $\left(v_{\max }, \mathrm{cm}^{-1}\right): 3390,3354,2960,1654,1582,1490,752$, 692. ${ }^{1} \mathrm{H}$ NMR (500 MHz, $\left.\mathrm{CDCl}_{3}\right)$ : $\delta \mathrm{H} 8.10$ (d, $\left.J_{4,6} 1.9 \mathrm{~Hz}, 1 \mathrm{H}, \mathrm{H}-6\right), 7.67$ (d, $J_{2 ", 3}$ " $=J_{5}$ ", 6 " $7.9 \mathrm{~Hz}$, 2H, H-2",6”), 7.50 (t, 2H, H-3",5”), 7.41 (t, J3",4"=J4",5" $7.5 \mathrm{~Hz}, 1 \mathrm{H}, \mathrm{H}-4$ "), 7.26 (t, $J_{3^{\prime}, 4^{\prime}=J_{4}, 5}, 7.2$ Hz, 1H, H-4'), 7.19 (d, J', , $^{\prime} 7.5$ Hz, 1H, H-6'), 7.15 (d, 1H, H-4), 6.89 (t, 1H, H-5'), 6.80 (d, 1H, H-3'), 3.90 (s, 2H, NH 2$).{ }^{13} \mathrm{C}$ NMR (125 MHz, CDCl $)$ : $\delta \mathrm{C} 160.0$ (C-3), 143.2 (C-2'), 141.3 (C1”), 138.1 (C-6), 134.8 (C-5), 131.0 (C-4'), 129.6 (C-6'), 128.8 (C-3”,5”), 128.2 (C-3”,5”), 128.2 (C-4,4”), 125.2 (C-2”,6”), 119.9 (C-1'), 119.7 (C-5'), 117.1 (C-3'). H” and C" are phenyl protons and carbons, resp. MS (ESI): $m / z$ (\%) 256, 261; HRMS (ESI) calcd. for $\mathrm{C}_{16} \mathrm{H}_{13} \mathrm{~N}_{3} \mathrm{O}$ $[\mathrm{M}+\mathrm{H}]^{+}:$264.1137, found: 264.1128 . 
5-(2-Azidophenyl)-2-phenylpyridazin-3(2H)-one (12c). 5-(2-Aminophenyl)-2-phenylpyridazin -3(2H)-one (11c) (2.00 g, $7.6 \mathrm{mmol})$ was dissolved in $37 \%$ hydrochloric acid $(60 \mathrm{~mL})$ and was cooled at $0^{\circ} \mathrm{C}$ with stirring. A solution of sodium nitrite $(1.11 \mathrm{~g}, 16.14 \mathrm{mmol})$ in water $(41 \mathrm{~mL})$ was added dropwise at such a rate that the temperature of the reaction mixture did not exceed 5 ${ }^{\circ} \mathrm{C}$. The mixture was stirred at this temperature for 1.5 hours. A solution of sodium azide (1.01 g, $16.14 \mathrm{mmol})$ and anhydrous sodium acetate $(8.73 \mathrm{~g}, 106.4 \mathrm{mmol})$ in water $(37 \mathrm{~mL})$ was then added at $0-5{ }^{\circ} \mathrm{C}$ and the mixture was stirred at this temperature for an additional 1 hour. Then the mixture was neutralized with a saturated sodium carbonate solution and extracted with dichloromethane $(3 \times 70 \mathrm{~mL})$. The organic layer was evaporated without heating and the residue was suspended with diethyl ether to yield brown crystals which were filtered off. The product decomposed on air and was therefore stored under argon atmosphere in a refrigerator. Brown crystals, yield 98\%, $2.15 \mathrm{~g} ; R_{\mathrm{f}}$ (chloroform-methanol 40:1): 0.68. IR ( $\left.v_{\max }, \mathrm{cm}^{-1}\right): 3057,2124$, 1670, 1488, 1292, 756, 720, 686. ${ }^{1} \mathrm{H}$ NMR (500 MHz, $\left.\mathrm{CDCl}_{3}\right): \delta \mathrm{H} 8.10$ (d, J4,6 $\left.2.2 \mathrm{~Hz}, 1 \mathrm{H}, \mathrm{H}-6\right)$, 7.68 (m, 2H, H-2", 6”), 7.54 (t, $J_{3}{ }^{\prime}, 4^{\prime}=J_{4}, 5^{\prime}, 8.0 \mathrm{~Hz}, 1 \mathrm{H}, \mathrm{H}-4^{\prime}$ ), 7.51 (m, 2H, H-3”, 5”), 7.42 (d, J5', , 7.0 Hz, 1H, H-6'), 7.42 (m, 1H, H-4”), 7.38 (d, 1H, H-3'), 7.29 (t, 1H, H-5'), 7.12 (d, 1H, H-4). ${ }^{13} \mathrm{C}$ NMR (125 MHz, $\mathrm{CDCl}_{3}$ ): $\delta \mathrm{C} 159.9$ (C-3), 141.8 (C-5), 141.3 (C-1”), 138.2 (C-6), 137.9 (C2'), 131.4 (C-4'), 130.4 (C-6'), 129.2 (C-4), 128.3 (C-3”,5’), 128.3 (C-4’), 126.0 (C-1'), 125.5 (C-5'), 125.3 (C-2",6”), 119.1 (C-3'). H” and C" are phenyl protons and carbons, resp.

\section{Compounds 13a-c were prepared according to Method B above.}

\section{5-(2-Triphenylphosphoranylideneaminophenyl)-2-methylpyridazin-3(2H)-one}

(13a). Yellow crystals, yield 84\%, $0.17 \mathrm{~g}, \mathrm{mp} \mathrm{81-82}{ }^{\circ} \mathrm{C} ; R_{\mathrm{f}}$ (ethyl acetate-chloroform 4:1): 0.51. IR $\left(v_{\max }, \mathrm{cm}^{-1}\right): 3854,3422,1654,1586,1472,1436,1338,1106,750,718,694 .{ }^{1} \mathrm{H}$ NMR $(500$ $\mathrm{MHz}_{\mathrm{CDCl}}$ ): $\delta_{\mathrm{H}} 8.42$ (d, J4,6 $\left.1.5 \mathrm{~Hz}, 1 \mathrm{H}, \mathrm{H}-6\right), 7.67$ (m, 6H, H-2",6”), 7.54 (m, 6H, H-3",5”),

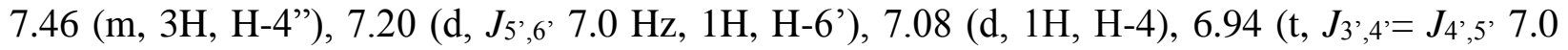

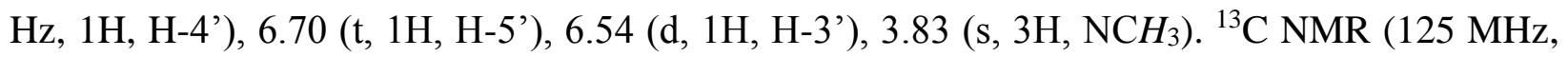
$\left.\mathrm{CDCl}_{3}\right): \quad \delta \mathrm{C} \quad 161.5$ (C-3), 149.2 (C-2'), $147.0 \quad(\mathrm{C}-5), 140.1 \quad(\mathrm{C}-6), \quad 132.5-128.5$ (C4',6',1",2",3",4",5",6”), 126.2 (C-4), 122.2 (C-3'), 117.6 (C-5'), $39.4\left(\mathrm{NCH}_{3}\right)$; H' and C' are phenyl protons and carbons, resp. of the phenylamino group; $\mathrm{H}^{\prime \prime}$ and $\mathrm{C}$ " are phenyl protons and carbons, resp. of the triphenylphosphoranylidene group. Anal. Calcd for $\mathrm{C}_{29} \mathrm{H}_{24} \mathrm{~N}_{3} \mathrm{OP} \times 1 / 2 \mathrm{H}_{2} \mathrm{O}$ (470.51): C, 74.03; H, 5.36; N, 8.93\%. Found: C, 74.41; H, 5.22; N, 8.70\%.

\section{5-(5-Chloro-2-triphenylphosphoranylideneaminophenyl)-2-methylpyridazin-3(2H)-one}

(13b). Yellow crystals, yield 80\%, $0.31 \mathrm{~g}, \mathrm{mp} 181.2-181.8{ }^{\circ} \mathrm{C} ; R_{\mathrm{f}}$ (chloroform-methanol 50:1): 0.20. IR ( $\left.v_{\max }, \mathrm{cm}^{-1}\right): 3052,1654,1582,1468,1436,1326,1108,806,720,694 .{ }^{1} \mathrm{H}$ NMR $(500$ MHz, DMSO-d6): $\delta$ н 8.29 (d, J4,6 $2.0 \mathrm{~Hz}, 1 \mathrm{H}, \mathrm{H}-6), 7.68$ (m, 6H, H-2”,6”), 7.63 (m, 6H, H-

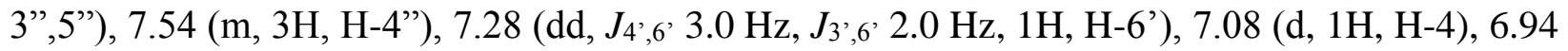
$\left(\mathrm{dd}, J_{3}{ }^{\prime}, 4^{\prime} 8.7 \mathrm{~Hz}, 1 \mathrm{H}, \mathrm{H}-4^{\prime}\right), 6.36\left(\mathrm{dd}, 1 \mathrm{H}, \mathrm{H}-3^{\prime}\right), 3.71\left(\mathrm{~s}, 3 \mathrm{H}, \mathrm{NCH}_{3}\right) .{ }^{13} \mathrm{C} \mathrm{NMR}(125 \mathrm{MHz}$, DMSO-d6): $\delta \mathrm{C} 159.9$ (C-3), 148.1 (C-2'), 144.7 (C-1'), 138.6 (C-6), 132.2 (C-4”), 132.0 (C2",6”), 129.6 (C-5), 129.5 (C-4'), 129.2 (C-1”), 129.0 (C-3”,5”), 128.9 (C-6'), 125.9 (C-4'), 122.5 (C-3'), 120.7 (C-5'), $39.0\left(\mathrm{NCH}_{3}\right)$. P-C coupling constants: ${ }^{1} J\left(\mathrm{P}, \mathrm{C}-1^{\prime \prime}\right) \quad 100 \mathrm{~Hz},{ }^{2} J(\mathrm{P}, \mathrm{C}-$ 2",6") $10 \mathrm{~Hz},{ }^{3} J\left(\mathrm{P}, \mathrm{C}-3\right.$ ", 5") $12 \mathrm{~Hz},{ }^{4} J(\mathrm{P}, \mathrm{C}-4 ") \quad 2.3 \mathrm{~Hz},{ }^{3} J\left(\mathrm{P}, \mathrm{C}-3\right.$ ') $11 \mathrm{~Hz},{ }^{4} J(\mathrm{P}, \mathrm{C}-6$ ') $1 \mathrm{~Hz}$; H' 


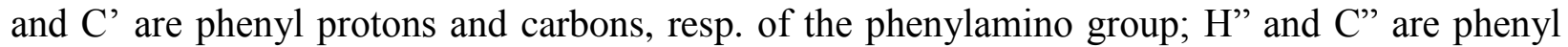
protons and carbons, resp. of the triphenylphosphoranylidene group. Anal. Calcd for $\mathrm{C}_{29} \mathrm{H}_{23} \mathrm{ClN}_{3} \mathrm{OP}$ (495.95): C, 70.23; H, 4.67; N, 8.47\%. Found: C, 70.27; H, 4.70; N, 8.35\%.

2-Phenyl-5-(2-triphenylphosphoranylideneaminophenyl)pyridazin-3(2H)-one (13c). Yellow oil, yield 85\%, $0.89 \mathrm{~g} ; R_{\mathrm{f}}$ (chloroform-methanol 40:1): 0.44. IR ( $\left.v_{\max }, \mathrm{cm}^{-1}\right): 3052,2924,1660$, 1586, 1472, 1434, 1336, 1106, 718, 692. ${ }^{1} \mathrm{H} \mathrm{NMR}\left(500 \mathrm{MHz}, \mathrm{CDCl}_{3}\right): \delta \mathrm{H} 8.56\left(\mathrm{~d}, J_{4,6} 8.0 \mathrm{~Hz}\right.$, 1H, H-6), 7.73 (m, 2H, H-2",6”), 7.54 (m, 3H, H-4"'), 7.51 (m, 2H, H-3",5”), 7.46 (m, 6H, H3"',5"'), 7.39 (m, 1H, H-4"), 7.30 (m, 6H, H-2"',6"'), 7.29 (m, 1H, H-6'), 7.15 (d, 1H, H-4), 6.99 (m, 1H, H-4'), 6.76 (m, 1H, H-5'), 6.58 (m, 1H, H-3'). ${ }^{13} \mathrm{C}$ NMR (125 MHz, CDCl ${ }_{3}$ ): extremely broadened $\delta \mathrm{c}$ signals. $\mathrm{H}^{\prime}$ and $\mathrm{C}$ " are phenyl protons and carbons, resp. of the $N$ phenyl group; H" and C", are phenyl protons and carbons, resp. of the triphenylphosphoranylidene group. MS (ESI): $\mathrm{m} / \mathrm{z}$ (\%) 520, 525; HRMS (ESI) calcd. for $\mathrm{C}_{34} \mathrm{H}_{26} \mathrm{~N}_{3} \mathrm{OP}[\mathrm{M}+\mathrm{H}]^{+}:$524.1892, found: 524.1897.

Method F. General procedure for the ring closure reaction of (13a-c) with phenylisocyanate The appropriate iminophosphorane $(1.00 \mathrm{mmol})$ 13a-c was dissolved in dry toluene $(20 \mathrm{~mL})$, purged with argon and was cooled to $0{ }^{\circ} \mathrm{C}$. Under stirring, a solution of phenylisocyanate $(0.15$ $\mathrm{mL}, 1.34 \mathrm{mmol})$ in toluene $(10 \mathrm{~mL})$ was added dropwise to the iminophosphorane at such a rate that the temperature of the reaction mixture did not exceed $5^{\circ} \mathrm{C}$. The mixture was stirred at this temperature for $1 \mathrm{~h}$. Then the mixture was refluxed at $140^{\circ} \mathrm{C}$ for 24 hours followed by evaporation in vacuo. After separation by column chromatography two products $\mathbf{1 4}$ and $\mathbf{1 5}$ were obtained.

3-Methyl-5-phenylamino-pyridazino[4,5-c]quinolin-4(3H)-one (14a). Yellow crystals, yield $10 \%, 0.04 \mathrm{~g}, 0.13 \mathrm{mmol}, \mathrm{mp} 178-181^{\circ} \mathrm{C} ; R_{\mathrm{f}}$ (ethyl acetate-chloroform 9:1): 0.85. IR $\left(v_{\max }, \mathrm{cm}^{-1}\right)$ : 3568, 3422, 3062, 2852, 1654, 1640, 1598, 1542, 744. ${ }^{1} \mathrm{H}$ NMR (500 MHz, DMSO-d $)$ : $\delta_{\mathrm{H}} 11.86$ (s, 1H, NH), 9.31 (s, 1H, H-1), 8.59 (d, $\left.J_{9,10} 8.3 \mathrm{~Hz}, 1 \mathrm{H}, \mathrm{H}-10\right), 8.03$ (d, $J_{2}, 3^{\prime}=J_{5}, 6^{\prime} 7.9 \mathrm{~Hz}, 2 \mathrm{H}$,

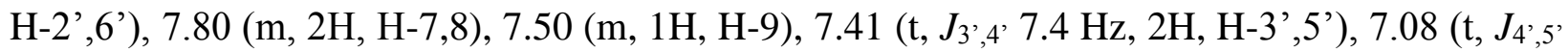
$7.4 \mathrm{~Hz}, 1 \mathrm{H}, \mathrm{H}-4$ '), 3.87 (s, 3H, N-CH 3 ). ${ }^{13} \mathrm{C}$ NMR (125 MHz, DMSO-d6): $\delta \mathrm{c} 159.8$ (C-4), 151.1 (C-5), 148.3 (C-6a), 139.7 (C-1'), 134.9 (C-4a), 134.0 (C-1), 132.6 (C-8), 128.9 (C-3',5'), 128.3 and 116.4 (C-10a and C-10b), 126.8 (C-7), 124.3 (C-10), 124.0 (C-9), 122.5 (C-4'), 119.5 (C2',6'), $39.5\left(\mathrm{~N}-\mathrm{CH}_{3}\right)$. H', C': are phenyl protons and carbons, resp. MS (ESI): $m / z$ (\%) 303, 274; HRMS (ESI) calcd. for $\mathrm{C}_{18} \mathrm{H}_{15} \mathrm{~N}_{4} \mathrm{O}[\mathrm{M}+\mathrm{H}]^{+}:$303.1246, found: 303.1252.

9-Chloro-3-methyl-5-phenylamino-pyridazino[4,5-c]quinolin-4(3H)-one $\quad(14 b)$ Yellow crystals, yield $23 \%, 0.07 \mathrm{~g}, 0.19 \mathrm{mmol}, \mathrm{mp} 259.5-260{ }^{\circ} \mathrm{C} ; R_{\mathrm{f}}$ (chloroform-methanol 40:1): 0.92. IR $\left(v_{\max }, \mathrm{cm}^{-1}\right): 3428,2924,1654,1614,1598,1540,1108,820,752 .{ }^{1} \mathrm{H}$ NMR $(500 \mathrm{MHz}$, $\mathrm{CF}_{3} \mathrm{COOD}$ ): $\delta \mathrm{H} 9.14$ (s, 1H, H-1), 8.44 (s, 1H, H-10), 7.86 (d, $\left.J_{7,8} 8.9 \mathrm{~Hz}, 1 \mathrm{H}, \mathrm{H}-8\right), 7.66$ (m, 2H, H-3',5'), 7.61 (m, 2H, H-7,4'), 7.49 (d, $J_{2}{ }^{\prime}, 3^{\prime}=J_{5}, 6^{\prime} 7.3 \mathrm{~Hz}, 2 \mathrm{H}, \mathrm{H}-2^{\prime}, 6^{\prime}$ ), 4.13 (s, 3H, N-CH3). ${ }^{13} \mathrm{C}$

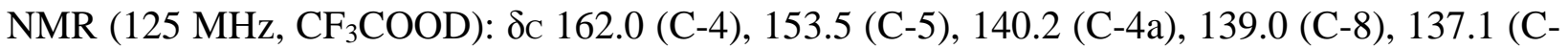
6a,9), 135.4 (C-1), 133.6 (C-3',5'), 133.4 (C-1'), 133.3 (C-4'), 128.0 (C-2',6'), 126.4 (C-10), $122.0(\mathrm{C}-7), 118.8(\mathrm{C}-10 \mathrm{a}), 112.8(\mathrm{C}-10 \mathrm{~b}), 42.8\left(\mathrm{~N}-\mathrm{CH}_{3}\right)$. H'and $\mathrm{C}^{\prime}$ are phenyl protons and 
carbons, resp. MS (ESI): $m / z(\%)$ 337, 308; HRMS (ESI) calcd. for $\mathrm{C}_{18} \mathrm{H}_{14} \mathrm{ClN}_{4} \mathrm{O}[\mathrm{M}+\mathrm{H}]^{+}$: 337.0856, found: 337.0844 .

3-Phenyl-5-phenylamino-pyridazino[4,5-c]quinolin-4(3H)-one (14c). Yellow crystals, yield $20 \%, 0.08 \mathrm{~g}, 0.21 \mathrm{mmol}, \mathrm{mp} 189-190{ }^{\circ} \mathrm{C} ; R_{\mathrm{f}}$ (chloroform-ethylacetate 10:1): 0.88. IR ( $v_{\max }, \mathrm{cm}^{-}$ $\left.{ }^{1}\right):$ 3426, 2924, 1724, 1658, 1602, 1578, 1552, 1448, 1314, 756, 688. ${ }^{1} \mathrm{H}$ NMR (500 MHz, DMSO-d6): $\delta \mathrm{H} 11.70$ (s, 1H, NH), 9.46 (s, 1H, H-1), 8.64 (d, J9,10 $8.2 \mathrm{~Hz}, 1 \mathrm{H}, \mathrm{H}-10), 8.02$ (d,

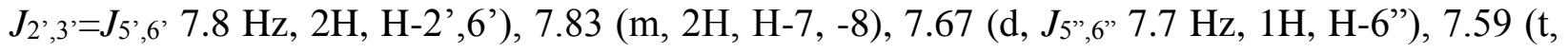
$J_{4}, 5^{\prime} 7.5 \mathrm{~Hz}, 1 \mathrm{H}, \mathrm{H}-5^{\prime \prime}$ ), 7.52 (m (overlapping), 2H, H-9,4”), 7.40 (t, 2H, H-3',5'), 7.08 (t, $\left.J_{3}, 4^{\prime}=J_{4}, 5^{\prime}, 7.4 \mathrm{~Hz}, 1 \mathrm{H}, \mathrm{H}-4^{\prime}\right)$. ${ }^{13} \mathrm{C}$ NMR (125 MHz, DMSO-d6): $\delta \mathrm{c} 159.8$ (C-4), 151.3 (C-5), 148.6 (C-6a), 141.3 (C-1'), 139.6 (C-1’'), 134.9 (C-10b), 134.8 (C-1), 132.9 (C-8), 128.9 (C3”,5’), 128.8 (C-3',5'), 128.4 (C-9), 126.9 (C-7), 126.3 (C-2',6'), 124.4 (C-10), 124.2 (C-4'), 122.6 (C-4”), 119.6 (C-2”,6”), 116.4 (C-10a), 110.0 (C-4a). H', C': $N^{3}$ - phenyl protons; H” and C": $N$-5-phenyl protons and carbons, resp. of the phenylamino group. MS (ESI): $m / z(\%) 272$, 365; HRMS (ESI) calcd. for $\mathrm{C}_{23} \mathrm{H}_{16} \mathrm{~N}_{4} \mathrm{O}[\mathrm{M}+\mathrm{H}]^{+}: 365.1402$, found: 365.1397.

5-[2-(1,6-Dihydro-1-methyl-6-oxopyridazin-4-yl)-phenylamino]-3-methylpyridazino[4,5-c]quinolin-4(3H)-one (15a). Yellow crystals, yield 45\%, $0.12 \mathrm{~g}, 0.29 \mathrm{mmol}, \mathrm{mp} \mathrm{274-275}{ }^{\circ} \mathrm{C} ; R_{\mathrm{f}}$ (ethyl acetate-chloroform 9:1): 0.36. IR $\left(v_{\max }, \mathrm{cm}^{-1}\right)$ : 3445, 1651, 1583, 1542, 1401, 1189, 1117 , 758. ${ }^{1} \mathrm{H}$ NMR (400 MHz, CF 3 COOD): $\delta \mathrm{H} 9.22$ (s, 1H, H-1), 8.62 (s, 1H, H-3”), 8.50 (d, J9,10 8.3 Hz, 1H, H-10), 7.97 (t, $\left.J_{7,8}=J_{8,9} 8.1 \mathrm{~Hz}, 1 \mathrm{H}, \mathrm{H}-8\right), 7.88-7.75$ (m, 6H, H-9,3',4',5',6',5’), 7.65 (d,

$1 \mathrm{H}, \mathrm{H}-7), 4.08(\mathrm{~s}, 3 \mathrm{H})$ and $4.05(\mathrm{~s}, 3 \mathrm{H}):\left(N^{3}-\mathrm{CH}_{3}\right.$ and $\left.N^{1}-{ }^{\prime} \mathrm{CH}_{3}\right) .{ }^{13} \mathrm{C} \mathrm{NMR}(100 \mathrm{MHz}$, $\mathrm{CF}_{3} \mathrm{COOD}$ ): $\delta \mathrm{c} 166.4$ (C-4), 164.2 (C-6”), 156.1 (C-5), 149.1 (C-4”), 146.2 (C-3”), 143.2 (C10b), 140.9 (C-8), 140.6 (C-6a), 137.9 (C-5'), 137.7 (C-1), 135.9 (C-3',4'), 135.5 (C-1'), 134.1 (C-2'), 132.5 (C-9), 131.7 (C-6’), 130.6 (C-5’), 129.0 (C-10), 122.8 (C-7), 120.2 (C-10a), 114.3 (C-4a), 45.0 and 44.6: $\left(N^{1}-{ }^{\prime} \mathrm{CH}_{3}\right.$ and $\left.N^{3}-\mathrm{CH}_{3}\right)$. $\mathrm{H}^{\prime}$ and $\mathrm{C}^{\prime}$ are phenyl protons and carbons, resp.; H" and C" are pyridazinyl protons and carbons and carbons, resp. MS (ESI): $m / z(\%)$ 199, 212, 411. Anal. Calcd for $\mathrm{C}_{23} \mathrm{H}_{18} \mathrm{~N}_{6} \mathrm{O}_{2}$ (410.43): C, 67.31; H, 4.42; N, 20.48\%. Found: C, 67.00; H, $4.38 ; \mathrm{N}, 20.39 \%$.

5-[2-(1,6-Dihydro-1-methyl-6-oxopyridazin-4-yl)-4-chlorophenyl-amino]-9-chloro-3-methyl -pyridazino[4,5-c] quinolin-4(3H)-one (15b). Yellow crystals, yield 49\%, $0.10 \mathrm{~g}, 0.20 \mathrm{mmol}$, mp 316.8-317.2 ${ }^{\circ} \mathrm{C} ; R_{\mathrm{f}}$ (chloroform-methanol 40:1): 0.62. IR $\left(v_{\max }, \mathrm{cm}^{-1}\right): 3422,3070,1654$, 1582, 1562, 1534, 1406, 1330, 826, 708. ${ }^{1} \mathrm{H}$ NMR (500 MHz, $\mathrm{CF}_{3} \mathrm{COOD}$ ): $\delta \mathrm{H} 9.15$ (s, 1H, H-1), 8.52 (s, 1H, H-3”), 8.48 (s, 1H, H-10), 7.91 (d, J7,8 9.0 Hz, 1H, H-8), 7.80 (d, J5,, 9.0 Hz, 1H, H5'), 7.79 (s, 1H, H-5’), 7.73 (s, 1H, H-3'), 7.72 (d, 1H, H-6'), 7.64 (dd, J7,10 1.6 Hz, 1H, H-7), $4.07(\mathrm{~s}, 3 \mathrm{H})$ and $4.02(\mathrm{~s}, 3 \mathrm{H}):\left(N^{3}-\mathrm{CH}_{3}\right.$ and $\left.N^{l}{ }^{\prime \prime}-\mathrm{CH}_{3}\right) .{ }^{13} \mathrm{C} \mathrm{NMR}\left(125 \mathrm{MHz}, \mathrm{CF}_{3} \mathrm{COOD}\right): \delta \mathrm{C}$ 167.2 and 164.2 (C-4 and C-6”), 156.4 (C-5), 147.5 (C-4”), 145.3 (C-3”), 143.0 (C-4'), 142.4 (C10b), 141.3 (C-8), 139.8 (C-6a), 139.3 (C-9), 137.8 (C-5'), 137.6 (C-1'), 137.5 (C-1), 136.0 (C3'), 133.4 (C-6'), 132.6 (C-2'), 131.8 (C-5’), 128.7 (C-10), 124.2 (C-7), 121.6 (C-10a), 115.0 (C-4a), $45.0\left(\mathrm{~N}^{3}-\mathrm{CH}_{3}, \mathrm{~N}^{1}\right.$ " $\left.-\mathrm{CH}_{3}\right)$; H' and C' are phenyl protons and carbons, resp.; H" and C": are pyridazinyl protons and carbons, resp. Anal. Calcd for $\mathrm{C}_{23} \mathrm{H}_{16} \mathrm{Cl}_{2} \mathrm{~N}_{6} \mathrm{O}_{2}$ (479.33): C, 57.63; $\mathrm{H}$, 3.36; N, 17.53; Cl, 14.79\%. Found: C, 57.87; H, 3.43; N, 17.20, Cl, 14.77\%. 
5-[2-(1,6-Dihydro-6-oxo-1-phenylpyridazin-4-yl)-phenylamino]-3-phenylpyridazino[4,5-c]quinolin-4(3H)-one (15c). Yellow crystals, yield 52\%, $0.15 \mathrm{~g}, 0.27 \mathrm{mmol}, \mathrm{mp} \mathrm{310-311}{ }^{\circ} \mathrm{C} ; R_{\mathrm{f}}$ (chloroform-ethyl acetate 10:1): 0.19. IR $\left(v_{\max }, \mathrm{cm}^{-1}\right): 3424,3058,2924,1662,1648,1588,1568$, 1536, 1450, 756, 684. ${ }^{1} \mathrm{H}$ NMR (500 MHz, CF $\mathrm{COOD}_{3}$ ): $\delta \mathrm{H} 9.3$ (s, 1H, H-1), 8.53 (J3",5" $1.9 \mathrm{~Hz}$, $1 \mathrm{H}, \mathrm{H}-3$ ”), 8.50 (d, J9,10 $8.3 \mathrm{~Hz}, 1 \mathrm{H}, \mathrm{H}-10), 7.95$ (t, $\left.J_{7,8}=J_{8,9} 7.9 \mathrm{~Hz}, 1 \mathrm{H}, \mathrm{H}-8\right), 7.78$ (s, 1H, H-5”), 7.77 (s, 1H, H-9), 7.73-7.80 (overlapping, 4H, H-3',4',5',6'), 7.67 (d, $J_{7,9} 7.9$ Hz, 1H, H-7), 7.307.60 (overlapping, $10 \mathrm{H}, \mathrm{Ph}-\mathrm{H}) .{ }^{13} \mathrm{C}$ NMR (125 MHz, $\left.\mathrm{CF}_{3} \mathrm{COOD}\right): \delta \mathrm{c} 165.3$ and $164.5(\mathrm{C}-4$ and C-6”), 156.5 (C-5), 148.6 (C-4”), 145.4 (C-3”), 143.8 (C-10b), 143.1 and 143.0 (Ph-C-1"' and Ph-C-1'”'), 141.4 (C-8), 141.2 (C-6a), 138.6 (C-1), 137.9 (C-5'), 136.0 (C-3',4'), 135.5 (C-1'),

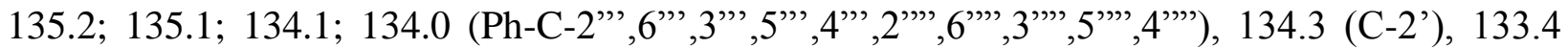
(C-6'), 132.9 (C-9), 131.4 (C-5”), 129.3 (C-10), 122.9 (C-7), 120.5 (C-10a), 115.1 (C-4a). H”": $N^{3}$-phenyl protons; H"': $N^{1}$ "'-phenyl protons. MS (ESI): $\mathrm{m} / \mathrm{z}$ (\%) 536, 541; HRMS (ESI) calcd. for $\mathrm{C}_{33} \mathrm{H}_{22} \mathrm{~N}_{6} \mathrm{O}_{2}[\mathrm{M}+\mathrm{H}]^{+}:$535.1882, found: 535.1884 .

$N$-[2-(1,3-Dimethyl-2,4-dioxo-1,2,3,4-tetrahydropyrimidine-5-yl)phenyl]pivalamide (17). 5Bromo-1,3-dimethylpyrimidine-2,4(1H,3H)-dione $\quad \mathbf{1 6}(0.44 \quad \mathrm{~g}, 2.00 \mathrm{mmol})$ and tetrakis(triphenylphosphine)-palladium $(0)(0.12 \mathrm{~g}, 0.10 \mathrm{mmol})$ as a catalyst were dissolved in dimethoxyethane $\left(12 \mathrm{~mL}\right.$, distilled over $\left.\mathrm{SnCl}_{2}\right)$ and were stirred at room temperature under argon for $30 \mathrm{~min}$. Pivaloylamino-phenylboronic acid $(0.71 \mathrm{~g}, 3.20 \mathrm{mmol})$ and sodium carbonate solution $(2 \mathrm{M}, 1.0 \mathrm{~mL})$ were then added and the mixture was refluxed for 8 hours. The reaction mixture was poured onto ice-water $(30 \mathrm{~mL})$ and was extracted with chloroform $(3 \times 30 \mathrm{~mL})$. Evaporation of the organic layer gave a crude product which was recrystallized. Colourless crystals, yield $91 \%, 0.57 \mathrm{~g}, \mathrm{mp} 165-166{ }^{\circ} \mathrm{C}$ (from ethyl acetate); $R_{\mathrm{f}}$ (chloroform-ethyl acetate 1:1): 0.63. IR ( $\left.v_{\max }, \mathrm{cm}^{-1}\right): 3286,2954,1704,1676,1638,1576,1510,1440,1356,1296,1164$, 924, 786, 750, 688. ${ }^{1} \mathrm{H}$ NMR (200 MHz, $\mathrm{CDCl}_{3}$ ): $\delta \mathrm{H} 8.63$ (s, 1H, NH), 7.72 (d, J3,4 $8.0 \mathrm{~Hz}, 1 \mathrm{H}$, H-3), 7.47-7.03 (m, 4H, H-6', 4,5,6), 3.47 (s, 3H) and $3.46(\mathrm{~s}, 3 \mathrm{H}):\left(N^{1}-\mathrm{CH}_{3}\right.$ and $\left.N^{3}-\mathrm{CH}_{3}\right), 1.21$ (s,

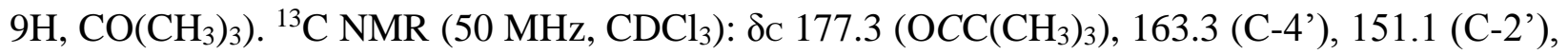
143.8 (C-6'), 136.7 (C-1), 130.0 (C-3), 129.1 (C-4), 127.0 (C-2), 126.3 (C-5), 125.5 (C-6), 113.2

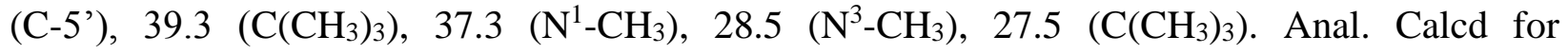
$\mathrm{C}_{17} \mathrm{H}_{21} \mathrm{~N}_{3} \mathrm{O}_{3}$ (315.37): C, 64.74; H, 6.71; N, 13.32\%. Found: C, 64.82; H, 6.69; N, 13.34\%.

5-(2-Aminophenyl)-1,3-dimethylpyrimidine-2,4(1H,3H)-dione (18). $N$-[2-(1,3-Dimethyl-2,4dioxo-1,2,3,4-tetrahydropyrimidine-5-yl)phenyl]pivalamide 17 (0.27 g, $0.87 \mathrm{mmol})$ was added to sulfuric acid $(20 \%, 20 \mathrm{~mL})$ and the mixture was refluxed for 3 hours. After cooling, the $\mathrm{pH}$ of the mixture was adjusted to $\mathrm{pH} 8$ by addition of aqueous sodium hydroxide $(40 \%)$. The mixture was then extracted with chloroform $(3 \times 30 \mathrm{~mL})$ and the crude product obtained after evaporation of the organic layer was recrystallized. Pale brown crystals, yield 88\%, $0.18 \mathrm{~g}, \mathrm{mp} 190.0-191.0$ ${ }^{\circ} \mathrm{C}$ (from chloroform-petroleum ether $\left.1: 1\right) ; R_{\mathrm{f}}$ (chloroform-ethyl acetate $\left.1: 1\right): 0.34$. IR $\left(v_{\max }, \mathrm{cm}^{-}\right.$ $\left.{ }^{1}\right)$ : 3396, 3328, 1696, 1646, 1620, 1494, 1448, 1352, 1298, 780, 754, 690. ${ }^{1} \mathrm{H}$ NMR (400 MHz,

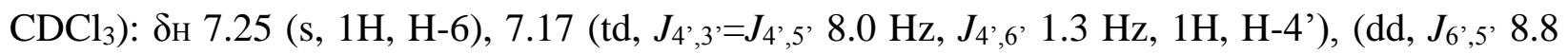
Hz, 1H, H-6'), 6.78-6.74 (m, 2H, H-5', H-3'), 3.99 (s, 2H, NH2), 3.42 (s, 3H) and 3.41 (s, 3H): $\left(N^{1}-\mathrm{CH}_{3}\right.$ and $\left.N^{3}-\mathrm{CH}_{3}\right) .{ }^{13} \mathrm{C} \mathrm{NMR}\left(100 \mathrm{MHz}, \mathrm{CDCl}_{3}\right)$ : $\delta \mathrm{C} 163.0(\mathrm{C}-4), 152.1(\mathrm{C}-2), 146.6(\mathrm{C}-2$ '), 
143.4 (C-6), 131.5 (C-6'), 130.2 (C-4'), 120.7 (C-1'), 119.7 (C-5'), 117.9 (C-3'), 114.2 (C-5), $37.7\left(N^{1}-\mathrm{CH}_{3}\right), 29.0\left(N^{3}-\mathrm{CH}_{3}\right)$. Anal. Calcd for $\mathrm{C}_{12} \mathrm{H}_{13} \mathrm{~N}_{3} \mathrm{O}_{2}(231.25)$ : C, 62.33; H, 5.67; N, $18.17 \%$. Found: $\mathrm{C}, 61.73 ; \mathrm{H}, 5.58 ; \mathrm{N}, 18.05 \%$.

5-(2-Azidophenyl)-1,3-dimethylpyrimidine-2,4(1H,3H)-dione (19). Prepared from 18 according to the procedure given for 12c. Yellow crystals, yield $96 \%, 0.34 \mathrm{~g} ; R_{\mathrm{f}}$ (chloroformethyl acetate 10:1): 0.27. IR $\left(v_{\max }, \mathrm{cm}^{-1}\right): 3068,2928,2116,1704,1654,1632,1574,1496,1444$, 1348, 930, 784, 750. ${ }^{1} \mathrm{H}$ NMR (400 MHz, $\mathrm{CDCl}_{3}$ ): $\delta \mathrm{H} 7.40$ (m, 1H, H-4'), 7.29 (dd, $J_{5}, 6,8.0 \mathrm{~Hz}$, $J_{4}^{\prime}, 6^{\prime} 1.6 \mathrm{~Hz}, 1 \mathrm{H}, \mathrm{H}-6^{\prime}$ ), 7.23 (s, 1H, H-6), 7.22 (dd, $J_{3}{ }^{\prime}, 4^{\prime} 8.0 \mathrm{~Hz}, J_{3}, 5^{\prime}, 0.8 \mathrm{~Hz}, 1 \mathrm{H}, \mathrm{H}-3^{\prime}$ '), 7.17 (td, $J_{4}, 5^{\prime} 8.0 \mathrm{~Hz}, 1 \mathrm{H}, \mathrm{H}-5$ '), $3.46\left(\mathrm{~s}, 3 \mathrm{H}, N^{1}-\mathrm{CH}_{3}\right), 3.41\left(\mathrm{~s}, 3 \mathrm{H}, N^{3}-\mathrm{CH}_{3}\right) .{ }^{13} \mathrm{C} \mathrm{NMR}(100 \mathrm{MHz}$, $\mathrm{CDCl}_{3}$ ): $\delta \mathrm{C} 162.7$ (C-4), 152.2 (C-2), 142.9 (C-6), 139.3 (C-2'), 132.7 (C-6’), 130.3 (C-4'), 125.4 (C-5'), 125.1 (C-1'), 119.2 (C-3'), $111.5(\mathrm{C}-5), 37.8\left(N^{1}-\mathrm{CH}_{3}\right), 28.9\left(N^{3}-\mathrm{CH}_{3}\right)$.

Compounds 20 and 22 were prepared according to method $\mathrm{B}$ above.

1,3-Dimethyl-5-(2-triphenylphosphoranylideneaminophenyl)pyrimidine-2,4(1H,3H)-dione (20). Yellow oil, yield 78\%, $0.46 \mathrm{~g} ; R_{\mathrm{f}}$ (toluene-methanol 4:1): 0.40. IR $\left(v_{\max }, \mathrm{cm}^{-1}\right): 3052,3006$, 2926, 1700, 1656, 1586, 1482, 1436, 1338, 1274, 750, 694. ${ }^{1} \mathrm{H}$ NMR (500 MHz, CDCl $): \delta_{\text {H }} 7.65$ (m, 6H, H-2",6"), 7.49 (m, 3H, H-4"), 7.41 (m, 6H, H-3",5”), 7.32 (s, 1H, H-6), 7.27 (m, 1H, H6'), 6.85 (td, $J_{4}{ }^{\prime},{ }^{\prime}=J_{5}, 6^{\prime} 7.4 \mathrm{~Hz}, J_{3}, 5^{\prime} 1.8 \mathrm{~Hz}, 1 \mathrm{H}, \mathrm{H}-5^{\prime}$ ), 6.69 (t, $J_{3^{\prime}, 4^{\prime}} 7.0 \mathrm{~Hz}, 1 \mathrm{H}, \mathrm{H}-4^{\prime}$ ), 6.52 (dt, $\left.J_{3}, 6^{\prime}, 1.0 \mathrm{~Hz}, 1 \mathrm{H}, \mathrm{H}-3^{\prime}\right), 3.36(\mathrm{~s}, 3 \mathrm{H})$ and $3.35(\mathrm{~s}, 3 \mathrm{H}):\left(N^{1}-\mathrm{CH}_{3}\right.$ and $\left.N^{3}-\mathrm{CH}_{3}\right) .{ }^{13} \mathrm{C}$ NMR $(125$ $\mathrm{MHz}, \mathrm{CDCl}_{3}$ ): $\delta \mathrm{C} 163.3$ (C-4), 152.7 (C-2), other signals are extremely broadened. H" are phenyl protons of the triphenylphosphoranylidene group. MS (ESI): $m / z$ (\%) 239, 279, 344, 388; HRMS (ESI) calcd. for $\mathrm{C}_{30} \mathrm{H}_{26} \mathrm{~N}_{3} \mathrm{O}_{2} \mathrm{P}[\mathrm{M}+\mathrm{H}]^{+}$: 492.1841, found: 492.1801 .

\section{1,3-Dimethyl-6-phenyl-5-(triphenylphosphoranylideneamino)pyrimidine-2,4(1H,3H)-dione}

(22). Yellow crystals, yield $82 \%, 0,62 \mathrm{~g}, \mathrm{mp} 217-218{ }^{\circ} \mathrm{C}$ (from dry ethanol); $R_{\mathrm{f}}$ (ethyl acetatechloroform 4:1): 0.50. IR ( $\left.v_{\max }, \mathrm{cm}^{-1}\right)$ : 3052, 1686, 1634, 1588, 1570, 1480, 1434, 1348, 1106,

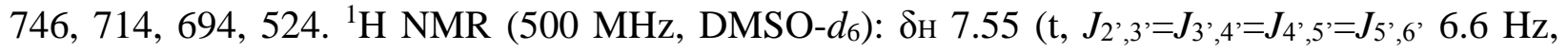
2H, H-3',5'), 7.52 (t, J4',5' 7.9 Hz, 1H, H-4'), 7.46 (m, 3H, H-4”), 7.45 (m, 6H, H-2”,6”), 7.42(d, 2H, H-2',6'), 7.36 (m, 6H, H-3", 5"), 3.08 (s, 3H, $\left.N^{3}-\mathrm{CH}_{3}\right), 3.00\left(\mathrm{~s}, 3 \mathrm{H}, N^{1}-\mathrm{CH}_{3}\right) .{ }^{13} \mathrm{C}$ NMR (125 MHz, DMSO-d6): $\delta$ c 162.3 (C-4), 149.8 (C-2), 137.4 (C-6), 134.7 (C-1',1”), 131.9 (C-2”,6”), 129.9 (C-2',6'), 128.7 (C-5), 128.4 (C-3',5',3”, 5”), 127.9 (C-4',4”), $34.4\left(N^{1}-\mathrm{CH}_{3}\right), 28.2\left(N^{3}-\right.$ $\mathrm{CH}_{3}$ ). P-C coupling constants: ${ }^{1} J\left(\mathrm{P}, \mathrm{C}-1\right.$ ") $102 \mathrm{~Hz},{ }^{2} J(\mathrm{P}, \mathrm{C}-2 ", 6 ") \quad 9.5 \mathrm{~Hz},{ }^{3} J(\mathrm{P}, \mathrm{C}-3 ", 5 ") \quad 11.7$ $\mathrm{Hz},{ }^{4} J(\mathrm{P}, \mathrm{C}-4$ ") $2.5 \mathrm{~Hz}$; H' and C' are phenyl protons and carbons, resp. of the phenyl group; $\mathrm{H}^{\prime \prime}$ and $\mathrm{C}$ " are phenyl protons and carbons, resp. of the triphenylphosphoranylidene group. Anal. Calcd for $\mathrm{C}_{30} \mathrm{H}_{26} \mathrm{~N}_{3} \mathrm{O}_{2} \mathrm{P} \times 1 / 2 \mathrm{C}_{2} \mathrm{H}_{5} \mathrm{OH}$ (514.56): C, 72.36; H, 5.68; N, 8.17\%. Found: C, 72.32; $\mathrm{H}, 5.22 ; \mathrm{N}, 8.09 \%$.

\section{$N$-[2-(1,3-Dimethyl-2,6-dioxo-1,2,3,6-tetrahydropyrimidin-4-yl)phenyl]- $N$ '-phenylthiourea}

(25). Compound $21(0.23 \mathrm{~g}, 1.00 \mathrm{mmol})$ was dissolved in dry acetonitrile $(10 \mathrm{~mL})$, then phenylisothiocyanate $(0.14 \mathrm{~g}, 0.12 \mathrm{ml}, 1.00 \mathrm{mmol})$ and 4-dimethylaminopyridine $(0.02 \mathrm{~g}, 0.17$ mmol) were added. The mixture was refluxed at $85^{\circ} \mathrm{C}$ under argon for 24 hours. The precipitated crystals were separated by filtration. Colourless crystals, yield $52 \%, 0.19 \mathrm{~g}, \mathrm{mp} 212-212.5^{\circ} \mathrm{C} ; R_{\mathrm{f}}$ (chloroform-methanol 40:1): 0.20. IR ( $\left.v_{\max }, \mathrm{cm}^{-1}\right)$ : 3280, 3130, 2964, 1694, 1652, 1534, 1508, 
1444, 1272, 762, 700. ${ }^{1} \mathrm{H}$ NMR (500 MHz, DMSO-d6): $\delta$ H 9.86 (s, 1H, NH-C-1"), 9.38 (s, 1H, NH-C-2'), 7.62 (dd, $J_{5}, 6^{\prime}, 7.9 \mathrm{~Hz}, J_{4}, 6^{\prime}, 0.9 \mathrm{~Hz}, 1 \mathrm{H}, \mathrm{H}-6$ '), 7.53 (td, $J_{4}, 5^{\prime}, 7.6 \mathrm{~Hz}, J_{3}, 5^{\prime}, 1.8 \mathrm{~Hz}, 1 \mathrm{H}$,

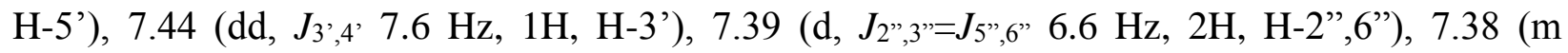
(overlapping), 1H, H-4'), 7.31 (t, $J_{4}$ ", 5" $7.5 \mathrm{~Hz}, 2 \mathrm{H}, \mathrm{H}-3$ ", 5"), 7.13 (t, J3",4" $7.3 \mathrm{~Hz}, 1 \mathrm{H}, \mathrm{H}-4$ "), $5.69(\mathrm{~s}, 1 \mathrm{H}, \mathrm{H}-5), 3.24\left(\mathrm{~s}, 3 \mathrm{H}, N^{3}-\mathrm{CH}_{3}\right), 3.07\left(\mathrm{~s}, 3 \mathrm{H}, N^{1}-\mathrm{CH}_{3}\right) .{ }^{13} \mathrm{C}$ NMR (125 MHz, DMSO-d $)$ :

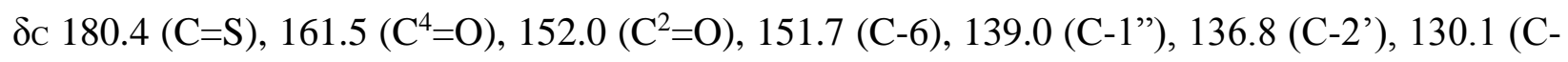
5'), 129.6 (C-1'), 129.3 (C-4'), 128.9 (C-6'), 128.5 (C-3”,5”), 126.2 (C-3'), 124.7 (C-4”), 123.5 (C-2",6"), $101.9(\mathrm{C}-5), 33.8\left(N^{l}-\mathrm{CH}_{3}\right), 27.5\left(N^{3}-\mathrm{CH}_{3}\right) . \mathrm{H}^{\prime}$ and $\mathrm{C}$ ": phenyl protons and carbons, resp. of the phenyl group at $\mathrm{S}=\mathrm{C}-\mathrm{NH}-\mathrm{Ph}$. MS (ESI): $\mathrm{m} / z$ (\%) 283, 327, 344, 388; HRMS (ESI) calcd. for $\mathrm{C}_{19} \mathrm{H}_{18} \mathrm{~N}_{4} \mathrm{O}_{2} \mathrm{~S}[\mathrm{M}+\mathrm{H}]^{+}: 367.1229$, found: 367.1240 .

\section{1,3-Dimethyl-5-phenylaminopyrimido[5,4-c]quinoline-2,4(1H, 3H)-dione (27). Compound 25}

$(0.30 \mathrm{~g}, 0.82 \mathrm{mmol})$ was dissolved in dichloromethane $(200 \mathrm{~mL})$, then triethylamine $(0.32 \mathrm{~g}$, $0.44 \mathrm{~mL}, 3.14 \mathrm{mmol})$ and 4-dimethylaminopyridine $(0.03 \mathrm{~g}, 0.23 \mathrm{mmol})$ were added. Methanesulfonylchloride $(0.61 \mathrm{~g}, 0.41 \mathrm{~mL}, 5.30 \mathrm{mmol})$ was dropped into the solution and the mixture was stirred at ambient temperature for 3 hours. Then the mixture was evaporated in vacuo and the obtained crude carbodiimide intermediate $\mathbf{2 6}$ was immediately dissolved in hot dry xylene $(10 \mathrm{~mL}$ of xylene/1 $\mathrm{g}$ of substrate). After 10 hours of reflux xylene was distilled off in vacuo and the crude product was purified by column chromatography and subsequent recrystallization. Yellowish brown crystals, yield 66\%, $0.18 \mathrm{~g}, \mathrm{mp} 208-208.5{ }^{\circ} \mathrm{C}$ (from methanol); $R_{\mathrm{f}}$ (chloroform-methanol 40:1): 0.77. IR $\left(v_{\max }, \mathrm{cm}^{-1}\right): 4400-1800$ (broad), 1700, 1660, 1596, 1548, 1352, 1042, 800, 754, 690. ${ }^{1} \mathrm{H}$ NMR (500 MHz, $\mathrm{CDCl}_{3}$ ): $\delta \mathrm{H} 11.26$ (s, 1H, NH), 8.01 (d, $\left.J_{9,10} 7.5 \mathrm{~Hz}, 1 \mathrm{H}, \mathrm{H}-10\right), 7.94$ (d, $J_{2}{ }^{\prime}, 3^{\prime}=J_{5}, 6^{\prime} 10.0 \mathrm{~Hz}, 2 \mathrm{H}, \mathrm{H}-2^{\prime}, 6^{\prime}$ ), 7.75 (d, $J_{7,8} 7.5 \mathrm{~Hz}, 1 \mathrm{H}, \mathrm{H}-$ 7), 7.62 (t, $\left.J_{8,9} 7.5 \mathrm{~Hz}, 1 \mathrm{H}, \mathrm{H}-8\right), 7.36$ (t, $J_{3}^{\prime}, 4^{\prime}=J_{5}, 6^{\prime}, 8.0 \mathrm{~Hz}, 2 \mathrm{H}, \mathrm{H}-3^{\prime}, 5^{\prime}$ ), 7.22 (t, 1H, H-9), 7.07 (t, 1H, H-4'), 3.91 (s, 3H, $\left.N^{1}-\mathrm{CH}_{3}\right), 3.50\left(\mathrm{~s}, 3 \mathrm{H}, N^{3}-\mathrm{CH}_{3}\right) .{ }^{13} \mathrm{C} \mathrm{NMR}\left(125 \mathrm{MHz}, \mathrm{CDCl}_{3}\right): \delta \mathrm{c} 163.9$ $\left(\mathrm{C}^{4}=\mathrm{O}\right), 152.9\left(\mathrm{C}^{2}=\mathrm{O}\right), 152.5,152.0$ and 150.9 (C-6a, C-10b and C-5), $140.6\left(\mathrm{C}-1^{\prime}\right), 133.1(\mathrm{C}-8)$, 129.4 (C-3',5'), 128.8 (C-7), 126.1 (C-10), 123.4 (C-4'), 122.5 (C-9), 121.3 (C-2',6'), 114.0 (C10a), 98.3 (C-4a), $41.5\left(N^{1}-\mathrm{CH}_{3}\right), 29.2\left(N^{3}-\mathrm{CH}_{3}\right)$. MS (ESI): $m / z(\%)$ 300, 327, 344, 388; HRMS (ESI) calcd. for $\mathrm{C}_{19} \mathrm{H}_{16} \mathrm{~N}_{4} \mathrm{O}_{2}[\mathrm{M}+\mathrm{H}]^{+}:$333.1352, found: 333.1353 .

2-(2-Triphenylphosphoranylideneaminophenyl)pyrazine (29). Prepared according to Method B above. Yellow crystals, yield $67 \%, 0.28 \mathrm{~g}, \mathrm{mp} 142.5-143.5{ }^{\circ} \mathrm{C} ; R_{\mathrm{f}}$ (toluene-methanol 4:1): 0.45. IR $\left(v_{\max }, \mathrm{cm}^{-1}\right): 3052,1656,1590,1484,1464,1436,1350,1298,716,694 .{ }^{1} \mathrm{H}$ NMR (500 $\left.\mathrm{MHz}, \mathrm{CDCl}_{3}\right)$ : $\delta \mathrm{H} 9.63(\mathrm{~m}, 1 \mathrm{H}, \mathrm{H}-3), 8.59$ (m, 1H, H-5), 8.38 (d, 1H, H-6), 7.68 (m, 7H, H6',2”,6”), 7.53 (m, 3H, H-4”), 7.45 (m, 6H, H-3”,5”), 6.97 (m, 1H, H-4'), 6.83 (m, 1H, H-5'), $6.63\left(\mathrm{~m}, 1 \mathrm{H}, \mathrm{H}-3\right.$ '). ${ }^{13} \mathrm{C} \mathrm{NMR}\left(125 \mathrm{MHz}, \mathrm{CDCl}_{3}\right)$ : extremely broadened $\delta \mathrm{c}$ signals. H" and C" are phenyl protons and carbons, resp. of the triphenylphosphoranylidene group. Anal. Calcd for $\mathrm{C}_{28} \mathrm{H}_{22} \mathrm{~N}_{3} \mathrm{P}$ (431.8): C, 77.94; H, 5.14; N, 9.74\%. Found: C, 77.76; H, 5.09; N, 9.60\%. MS (ESI): $m / z(\%) 388,409,476$; HRMS (ESI) calcd. for $\mathrm{C}_{28} \mathrm{H}_{22} \mathrm{~N}_{3} \mathrm{P}[\mathrm{M}+\mathrm{H}]^{+}: 432.1630$, found: 432.1637.

3-Phenylpyridazino[4,5-b]indole-4(3H)-one (30). A solution of 5-(2-azidophenyl)-2phenylpyridazin-3(2H)-one 12c $(0.73 \mathrm{~g}, 2.52 \mathrm{mmol})$ in dry xylene $(5 \mathrm{~mL})$ was refluxed for 24 hours, the solvent was then removed under reduced pressure and the residue was recrystallized. 
Pale brown crystals, yield 59\%, $0.39 \mathrm{~g}, \mathrm{mp} 323-324{ }^{\circ} \mathrm{C}$ (from isopropyl alcohol); $R_{\mathrm{f}}$ (toluenemethanol 4:1): 0.56. IR ( $\left.v_{\max }, \mathrm{cm}^{-1}\right): 3150,3086,3010,1656,1600,1532,1454,1308,760,738$. ${ }^{1} \mathrm{H}$ NMR (500 MHz, DMSO-d $\left.)_{6}\right): \delta_{\mathrm{H}} 12.91$ (s, $\left.1 \mathrm{H}, N^{5}-\mathrm{H}\right), 8.92$ (s, 1H, H-1), 8.21 (d, J8,9 8,0 Hz, 1H, H-9), 7.65 (d (overlapping), J6,7 $7.5 \mathrm{~Hz}, 1 \mathrm{H}, \mathrm{H}-6$; m, 2H, H-2',6'), 7.54 (m (overlapping),

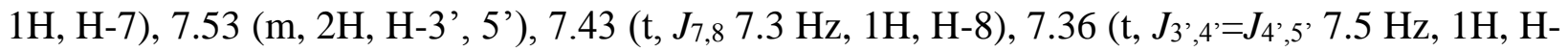
4'). ${ }^{13} \mathrm{C}$ NMR (125 MHz, DMSO- $\left.d_{6}\right)$ : $\delta \mathrm{c} 154.4$ (C-4), 142.0 (C-1'), 139.4 (C-5a), 133.7 (C-1), 131.7 (C-4a), 128.4 (C-3',5'), 127.5 (C-8), 127.1 (C-7), 126.2 (C-2',6'), 121.6 (C-4'), 121.5 (C9), 120.7 (C-9a), 116.9 (C-9b), 113.1 (C-6). H'and C' are phenyl protons and carbons, resp. Anal. Calcd for $\mathrm{C}_{16} \mathrm{H}_{11} \mathrm{~N}_{3} \mathrm{O} \times 1 / 2 \mathrm{H}_{2} \mathrm{O}$ (270.29): C, 71.10; H, 4.47; N, 15.55\%. Found: C, 71.37; $\mathrm{H}, 4.15$; N, 15.36\%. MS (ESI): $m / z$ (\%) 171, 219, 235, 262, 263; HRMS (ESI) calcd. for $\mathrm{C}_{16} \mathrm{H}_{11} \mathrm{~N}_{3} \mathrm{O}[\mathrm{M}+\mathrm{H}]^{+}:$262.0980, found: 262.0992 .

\section{Acknowledgements}

The authors are indebted to Pál Tapolcsányi, Nikolett Kállai and Ágnes Lernyei for their preparative work, to Benjámin Podányi and Tamás Gáti for recording of NMR spectra, as well as for helpful discussions, to the late Gyula Horváth and Caroline Meyers for the mass spectrometry measurements, to Péter Tétényi for recording the IR spectra and to Ágnes Puhr-Forgó for elementary analyses. Financial support of this work by Hungarian Scientific Research Fund (K73389), Medical Research Council (ETT 099-03/2009) and the National Development Agency (TÁMOP-4.2.1/B-09/1/KMR-2010-0001) is gratefully acknowledged.

\section{References}

1. Mátyus, P. J. Heterocycl. Chem. 1998, 35, 1075.

2. Mátyus, P.; Maes, B. U. W.; Riedl, Zs.; Hajós, Gy; Lemière, G. L. F.; Tapolcsányi, P.; Monsieurs, K.; Éliás, O.; Dommisse, R. A.; Krajsovszky, G. Synlett 2004, 7, 1123.

3. (a) Wamhoff, H.; Richardt, G.; Stoelben, S. Adv. Heterocycl. Chem. 1995, 64, 159. (b) Eguchi, S.; Okano T., Okawa, T. In Recent Res. Devel. Org. Chem. Transworld Research Network Trivandrum; Pandalai: S. G. Wiley, 1997; Vol. 1. (c) Okawa, T.; Eguchi, S. Tetrahedron 1998, 54, 5853. (d) Alvarez, R.; Sarandes, Peinador C.; Quintela, J. M. Tetrahedron 2001, 57, 5413. (e) Pitterna, T.; Cassayre, J.; Huter, O. F.; Jung, P. M. J.; Maienfisch, P.; Kessabi, F. M.; Quaranta, L.; Tobler, H. Bioorg. Med. Chem. 2009, 17, 4085. (f) Alibes, R.; Figueredo, M. Eur. J. Org. Chem. 2009, 2421. (g) Lu, J-Y.; Riedrich, M.; Mikyna, M.; Arndt, H-D. Angew. Chem. Int. Ed. 2009, 48, 8137, S8137/1-S8137/10. (h) Xu, S-Z.; Cao, M-H.; Chen, C-S.; Ding, M-W. J. Heterocycl. Chem. 2009, 46, 903. (i) Mahdavi, H.; Amani, J. Tetrahedron Lett. 2009, 50, 5923. (j) He, P.; Wu, J.; Nie, Y-B.; Ding, M.-W. Tetrahedron 2009, 65, 8563. (k) Scondo, A.; Dumarcay-Charbonnier, F.; 
Barth, D.; Marsura, A. Tetrahedron Lett. 2009, 50, 5582. (1) Wang, H-Q.; Zhou, W-P.; Wang, Y-Y.; Lin, C-R.; Liu, Z-J. J. Heterocycl. Chem. 2009, 46, 256. (m) Porwanski, S.; Marsura, A. Eur. J. Org. Chem. 2009, 2047, S2047/1-S2047/3. (n) Beltran-Rodil, S.; Donald, J. R.; Edwards, M. G.; Raw, S. A.; Taylor, R. J. K. Tetrahedron Lett. 2009, 50, 3378. (o) Muthusamy, S.; Srinivasan, P. Tetrahedron Lett. 2009, 50, 1331. (p) Lorenzo, A.; Aller, E.; Molina, P. Tetrahedron 2009, 65, 1397. (q) Hirota S.; Sakai T.; Kitamura N.; Kubokawa K., Kutsumura N.; Otani T.; Saito T. Tetrahedron 2010, 66, 653.

4. Atkinson, C. M.; Rodway, R. E. J. Chem. Soc. 1959, 1.

5. Riedl, Zs.; Maes, B. U. W.; Monsieurs, K.; Lemière, G. L. F.; Mátyus, P.; Hajós, Gy. Tetrahedron 2002, 58, 5645.

6. Barlin, G. B.; Lakshminarayana, P. J. Chem. Soc., Perkin Trans. 1: Org. Bioorg. Chem. 1977, 1038.

7. Maes, B. U. W.; R'kyek, O.; Kosmrlj, J.; Lemière, G. L. F.; Esmans, E.; Rozenski, J.; Dommisse, R. A.; Haemers, A. Tetrahedron 2001, 57, 1323.

8. Krajsovszky, G.; Mátyus, P.; Riedl, Zs.; Csányi, D.; Hajós, Gy. Heterocycles 2001, 35,

9. 1105 .

10. (a) Kappe, T.; Pfaffenschlager, A.; Stadlbauer, W. Synthesis 1989, 9, 666. (b) Bader, J.;

11. Vogel, C. Ger. Offen. DE 2162046 (1972), Chem. Abstr. 1972, 77, 126668

12. Lapachev, V.V.; Stadlbauer, W.; Kappe, T. Monatsh. Chem. 1988, 119, 97.

13. Molina, P.; Conesa, C.; Velasco M. D. Synthesis 1996, 12, 1459.

14. Molina, P.; Fresneda, P. M.; Delgado, S. Synthesis 1999, 2, 326.

15. Godard, A.; Queguiner, G. J. Heterocycl. Chem. 1984, 21, 27.

16. Tapolcsányi, P.; Krajsovszky, G.; Andó, R.; Lipcsey, P.; Horváth, Gy.; Mátyus, P.; Riedl, Zs; Hajós, Gy.; Maes, B. U. W.; Lemière, G. L. F. Tetrahedron 2002, 58, 10137.

17. Mátyus, P.; Czakó, K.; Behr, Á.; Varga, I.; Podányi, B.; Von Arnim, M.; Várkonyi, P. Heterocycles 1993, 36, 785.

18. Éliás, O.; Károlyházy, L.; Stájer, G.; Fülöp, F.; Czakó, K.; Harmath, V.; Barabás, O.; Keserü, K.; Mátyus, P. J. Mol. Struct. 2001 545, 75.

19. (a) Mowry, D. T. J. Am. Chem. Soc. 1953, 75, 1909. (b) Terai T.; Azuma H.; Hattori R. Jap. Pat. 1300, 1967; Chem. Abstr. 1967, 66, 65497z.

20. Takaya, M. Yakugaku Zasshi 1988, 108, 911-915; Chem. Abstr. 1989, 110, 154250.

21. Krajsovszky, G.; Károlyházy, L.; Riedl, Zs.; Csámpai, A.; Dunkel, P.; Lernyei, Á.; DajkaHalász, B.; Hajós, Gy.; Mátyus P. J. Mol. Struct. 2005, 713, 235.

22. Coelho, A.; Sotelo, E.; Novoa, H.; Peeters, O. M.; Blaton, N.; Ravina, E. Tetrahedron 2004, 60, 12177.

23. Pilgram, K. H.; Pollard, G. E. J. Heterocycl. Chem. 1977, 14, 1039.

24. Yamasaki, T.; Kawaminami, E.; Yamada, T.; Okawara, T.; Furukawa, M. J. Chem. Soc., Perkin Trans. 1: Org. Bioorg. Chem. (1972-1999) 1991, 991.

25. Fischer, A.; Kropp, R.; Reicheneder, F. Ger. Offen. DE 1912770, 1970; Chem. Abstr. 1971, $74,53824 \mathrm{f}$. 
26. Kweon, D-H.; Kang, Y-J.; Chung, H-A.; Yoon, Y-J. J. Heterocycl. Chem. 1998, 35, 819.

27. Dajka-Halász, B.; Monsieurs, K.; Éliás, O.; Károlyházy, L.; Tapolcsányi, P.; Maes, B. U. W.; Riedl, Zs.; Hajós, Gy.; Dommisse, R. A.; Lemière, G. L. F.; Kosmrlj, J.; Mátyus, P. Tetrahedron 2004, 60, 2283.

28. Molina, P.; Vilaplana, M. J. Synthesis 1994, Special Issue, 1197-1218.

29. Jonckers, T. H. M.; Van Miert, S.; Cimanga, K.; Bailly, C.; Colson, P.; De Pauw-Gillet, MarieClaire; van den Heuvel, H.; Claeys, M.; Lemière, F.; Esmans, E.L.; Rozenski, J.; Quirijnen, L.; Maes, L.; Dommisse, R.; Lemiere, G. L. F.; Vlietinck, A.; Pieters, L. J. Med. Chem. 2002, 45, 3497.

30. Haede, W. J. Heterocycl. Chem. 1981, 18, 1417. 